\title{
A Computational and Experimental Study of Slit Resonators
}

\author{
C.K.W. Tam ${ }^{1}$ and H. Ju ${ }^{2}$ \\ Florida State University, Tallahassee, FL 32306-4510 \\ M.G. Jones ${ }^{3}$, W.R. Watson ${ }^{4}$ and T.L. Parrott ${ }^{5}$ \\ NASA Langley Research Center, Hampton, VA 23681-2199
}

\begin{abstract}
Computational and experimental studies are carried out to offer validation of the results obtained from direct numerical simulation (DNS) of the flow and acoustic fields of slit resonators. The test cases include slits with 90-degree corners and slits with 45-degree bevel angle housed inside an acoustic impedance tube. Three slit widths are used. Six frequencies from 0.5 to $3.0 \mathrm{kHz}$ are chosen. Good agreement is found between computed and measured reflection factors. In addition, incident sound waves having a white noise spectrum and a prescribed pseudo-random noise spectrum are used in a subsequent series of tests. The computed broadband results are again found to agree well with experimental data. It is believed the present results provide strong support that DNS can eventually be a useful and accurate prediction tool for liner aeroacoustics. The usage of DNS as a design tool is discussed and illustrated by a simple example.
\end{abstract}

\section{Introduction}

Nowadays, commercial jet engines are invariably fitted with acoustic liners for noise suppression purposes. Properly designed liners are effective in suppressing both tones and broadband noise. For nacelle liner applications, the local-reacting properties of perforate-honeycomb liners allow their acoustic behavior to be characterized by a single complex quantity called acoustic impedance. This quantity is greatly affected by high sound pressure levels and grazing flows. The lack of acoustic impedance models based on first principles necessitates a semiempirical approach to acoustic impedance prediction models. This semi-empiricism has been developed through extensive experimental tests and engineering experience over the years. Therefore, current prediction models are restricted in their applicability to innovative liner concepts by virtue of the limited validity range of the empirical parameters.

The simplest liner structure consists of a porous facesheet bonded to a honeycombcompartmented cavity. Multiple layers of these structures can be cascaded to attain broadband absorption by reducing the antiresonance resistance, such that they are nearly as effective as bulk absorbers. However, the design of optimized broadband liners poses a challenge because of the harsh aeroacoustic conditions in which the liners are required to operate and the consequent effect on liner acoustic impedance. The facesheet porosity is generally achieved with holes arranged in a regular pattern. The dimensions of the holes and cavities determine the range of frequencies over which the liner is most effective.

Because the effectiveness of such an absorber is sensitive to grazing flow speed and incident

\footnotetext{
${ }^{1}$ Robert O. Lawton Distinguished Professor, Department of Mathematics, Fellow AIAA.

${ }^{2}$ Post-Doctoral Research Associate, Department of Mathematics.

${ }^{3}$ Research Scientist, Structural Acoustics Branch, Aerodynamics, Aerothermodynamics and Acoustics Competency, Member AIAA.

${ }^{4}$ Senior Research Scientist, Computational Modeling and Simulation Branch, Aerodynamics, Aerothermodynamics and Acoustics Competency, Senior Member AIAA.

${ }^{5}$ Senior Research Scientist, Structural Acoustics Branch, Aerodynamics, Aerothermodynamics and Acoustics Competency.
} 
sound, it is regarded as weakly nonlinear. It is this nonlinearity that has complicated the design of acoustic liners for aircraft engine applications. Large databases have been collected over the past three decades for a range of relevant liner geometry and aeroacoustic parameters. Unfortunately, because of the small hole size, detailed experimental studies of the fluid dynamics associated with the aeroacoustic environment in the resonator cavity and in and around the holes have not been carried out. However, Ingard et al. ${ }^{1,2}$ conducted flow visualization studies and hot wire measurements on larger orifices. Their experimental observations, although not precisely matching the effective Reynolds number based on the hole diameter, became the basis of a number of semiempirical liner flow models. Physically, the effect of Reynolds number could be significant. Therefore, a careful and detailed study of the sound-induced flow field around the opening of a resonator at the correct Reynolds number would be extremely useful in identifying the dominant mechanism by which the resistive component of a resonant acoustic liner is related to the fluid/structure interaction.

Recently, Tam et al. ${ }^{3,4}$ initiated an effort to investigate the coupling between the flow and sound fields of resonant liners by direct numerical simulation (DNS). One advantage of DNS is that the smallness of the resonator opening is not a hindrance to the investigation, as is the case with experimentation, and that the correct viscous effect can be reproduced in the computation. In Ref [3], it was found that for slit resonators with slit widths of $1 \mathrm{~mm}$ or less the flow around the slit was laminar even at incident sound-pressure-level (SPL) as high as $155 \mathrm{~dB}$. The flow was totally dominated by vortex shedding. The vortices were shed from the corners of the resonator opening. This is quite different from the turbulent jet model considered by Melling $^{5}$ and used by others ${ }^{6,7}$ at high SPL. The vortex shedding process effectively transfers acoustic energy into kinetic energy associated with the rotational motion of the vortices. This rotational energy cannot be reverted back into acoustic energy. It is eventually dissipated into heat by molecular viscosity. By measuring and comparing the rate of energy transferred to the shed vortices and the energy dissipation rate due directly to the effect of viscosity in their numerical simulations, Tam et al. confirmed that vortex shedding was by far the dominant acoustic energy absorption mechanism in this regime.

The primary objective of the present investigation is to provide validation of DNS results. For this purpose, two series of slit resonator experiments were conducted with the NASA Langley Research Center normal incident impedance tube. In one series, the experiments used normal slits with 90-degree corners. In the other series, bevel slits of 45degree angles were employed. The acoustic impedances and absorption coefficients of resonators with slit widths of $0.05,0.1$ and 0.2 inches were measured using the twomicrophone method. ${ }^{8,9}$ Independently, direct numerical simulations of the same impedance tube experiments were performed at the Florida State University. Measurements and DNS calculations were conducted for six frequencies for each slit width, resulting in 36 cases altogether. The validity of DNS results is judged by comparing the measured and computed reflection factor for all 36 cases. It is worthwhile to point out that in Ref [4], partial validation of DNS results was achieved. However, in that investigation, the experiments were carried out in an impedance tube, but the numerical simulations were done without the tube. Because of this difference, only the measured and computed absorption coefficients of the resonators at different SPL and frequencies were compared and validated. In the present work, the same impedance tube was used in the experiments and simulations. Hence this may be considered one of the first times validation of the computed acoustic impedance of resonators by DNS has been successfully carried out to this level of detail. The authors recognize that studies of a single-slit plate mounted over a single cavity are quite different from realistic liners. However, it is crucial that this level of complexity be resolved with confidence before liners consisting of perforated plates (with numerous cylindrical holes) mounted onto 
honeycomb (multiple cavities) core can be successfully studied.

As a part of this effort, validation tests of DNS results of slit resonators under white noise excitation as well as under incident sound waves with a prescribed pseudorandom noise spectrum were also carried out. It was found that the computed resistance and reactance of the slit resonators over the entire frequency spectrum were in good agreement with measurements.

Another objective of this work is to demonstrate that DNS not only has the potential to improve our understanding of conventional acoustic liner flow physics, but may eventually be useful in liner design as well. To demonstrate that this expectation is realistic, we apply DNS to the investigation of orifice flow field changes arising from modifying the lip geometry of a slit resonator. Such changes are expected to have an impact on the sound absorption ability of the resonator. It was mentioned above that vortex shedding at the opening of a resonator plays an important role in the resistive component of acoustic impedance and consequently the absorption of incident acoustic energy. To achieve maximum absorption, the acoustic impedance must be properly matched to the source field. Thus, to improve sound absorption, a specific value of resistance (and therefore vortex shedding, if that is the dominant resistance contributor) must be implemented. It is important therefore to understand the effect of small modifications in orifice geometry on acoustic impedance. One simple modification is to replace normal slits (90-degree corners) with bevel slits (acute-angle corners). To test this geometrical modification in liner design, a series of numerical simulations of the performance of slit resonators in a normal incidence impedance tube is carried out. Visualization of the computed flow field reveals that at low SPL there is no vortex shedding in the case of a normal slit, but vortex shedding occurs in the case of a bevel slit. As expected, this increase in vortex shedding results in an increase in resistance. This example illustrates the possibility of using DNS to provide information that could be useful to the choice and design of acoustic liner resonators.

\section{Experimental Instrumentation and Procedure}

\section{A. Impedance Tube Description}

The data used in the current study were acquired in a normal incidence tube (NIT) located in the Flow Impedance Test Laboratory of NASA Langley Research Center. A schematic illustrating this apparatus is shown in figure 1 . The tube has a cross-section of 2-in by 2 -in and a length of 24 inches. The test specimen surface is made to be coincident with the exit plane of the NIT by means of a clamping arrangement designed to achieve an airtight fit. Six 120-watt acoustic drivers are combined via a manifold to generate acoustic plane waves over a frequency range of 0.5 to $3.0 \mathrm{kHz}$, with sound pressure levels (SPL's) up to $155 \mathrm{~dB}$ at the test specimen surface. The acoustic waves interact with the test specimen to create a standing wave pattern that uniquely characterizes the normal incidence acoustic impedance of the test specimen.

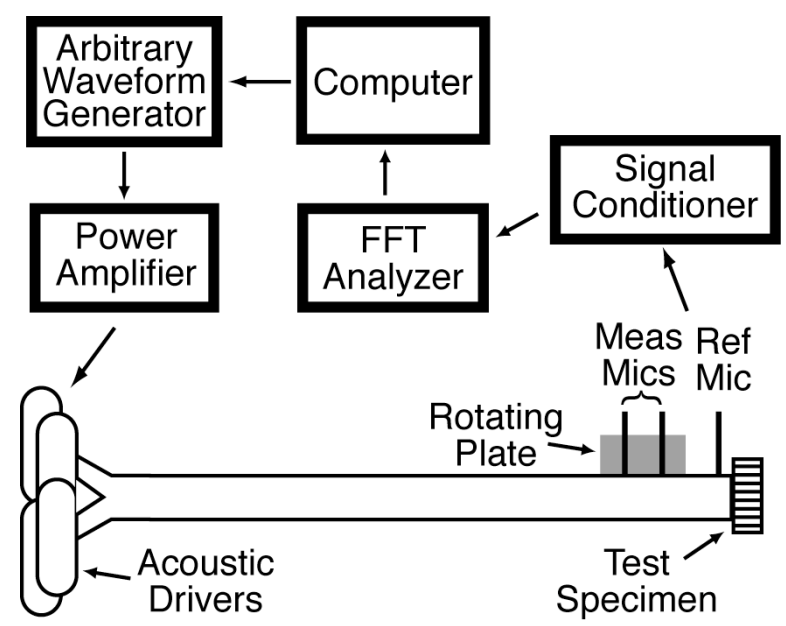

Figure 1. NASA LaRC Normal Incidence Tube with instrumentation.

An 0.25-in-diameter condenser-type reference microphone (see figure 1), flush mounted 0.25 inches from the test specimen surface in the top wall of the NIT, is used to determine the total (incident plus reflected) SPL at the test specimen surface. The Two-Microphone Method $^{9}$ (TMM) is implemented with two microphones flush-mounted 1.25 inches apart in a rotating steel plate in the top wall. This allows the positions of two 0.25 in-diameter 
condenser-type microphones to be switched in a very convenient and precise manner. The acoustic impedance of the test specimen, determined using the TMM, can then be used to separate the total acoustic pressure into incident and reflected acoustic pressures.

\section{B. Acoustic Source Description}

Three types of acoustic source were used. The first was a single discrete frequency source. A function generator was used to generate discrete frequencies over the frequency range of 0.5 to $3.0 \mathrm{kHz}$, in steps of $0.5 \mathrm{kHz}$. At each frequency, the amplitude was set to the maximum achievable level (up to $155 \mathrm{~dB}$ ) at the reference microphone location. For these tests, the harmonic content was at least $10 \mathrm{~dB}$ below the SPL recorded at the fundamental frequency.

The second acoustic source was a broadband noise source. A white-noise generator was used to generate a broadband sound field at an overall sound pressure level (integrated from 0.5 to $3.0 \mathrm{kHz}$ ) of $140 \mathrm{~dB}$ in the normal incidence tube. The resultant SPL's and phases at each of the microphone locations were recorded at $25 \mathrm{~Hz}$ increments from 0.5 to $3.0 \mathrm{kHz}$.

The third acoustic source was pseudorandom noise. This noise source was composed of a combination of thirteen discrete frequencies, with prescribed amplitudes and phases at each frequency. To accomplish this, a computer code generated a digitized pattern as input to an arbitrary waveform generator. The analog signal created from this digitized pattern was repetitively output from the waveform generator. By adjusting amplitude and phase parameters in the computer code (performed automatically by the code), the amplitude of each constituent frequency at the reference microphone location was adjusted until the incident SPL of each was within $\pm 1.0 \mathrm{~dB}$ of the target amplitude $(136 \mathrm{~dB})$. This procedure has the advantage of control of the incident sound spectrum while maintaining a broadband noise distribution.

The incident acoustic pressures produced by the discrete frequency and pseudo-random noise source types were used as input to the computational method (DNS) described elsewhere in this paper to compute the acoustic impedance of the test specimen. Comparisons of the measured and computed acoustic impedances (or related quantities) are used to demonstrate the validity of the computational method.

\section{Electronic Instrumentation}

Figure 1 also shows the instrumentation used in the acoustic impedance measurements. An arbitrary waveform generator (AWG) is used to output the electronic signals to generate either acoustic source type. For each test, a Fast Fourier Transform (FFT) analyzer is used to measure two transfer functions between the outputs of the microphones in the rotatingplate (one with plate at zero degrees and one with plate rotated 180 degrees). These results are stored on the computer. Any effects of mismatched responses between the two microphone channels are removed by appropriately averaging the two transfer functions. As mentioned earlier, this switched-microphone implementation of the TMM allows the acoustic impedance of the test specimen to be conveniently determined with confidence.

\section{Description of Test Specimens}

As shown in figure 2, the six test specimens consisted of single slits centered in 2 insquare, 0.04 in-thick facesheets that capped an airtight 2 in-square cross-section cavity, 6 inches deep. One objective of this test was to determine the effect of a $45^{\circ}$ bevel (linearly flared slit walls) on the acoustic impedance relative to that measured with no bevel (parallel slit walls). The span-wise dimension of each slit was held constant at 2 inches to match the width of the impedance tube. For the three baseline (parallel wall) slits, the slit widths (slit wall separations) were $0.05,0.10$ and 0.20 inches. The minimum widths for the three corresponding beveled slits were set to the same dimensions. Finally, the same backing cavity geometry was used for each test specimen to insure that resonance frequency differences between each of the slit specimens would be completely attributable to slit geometry effects. 


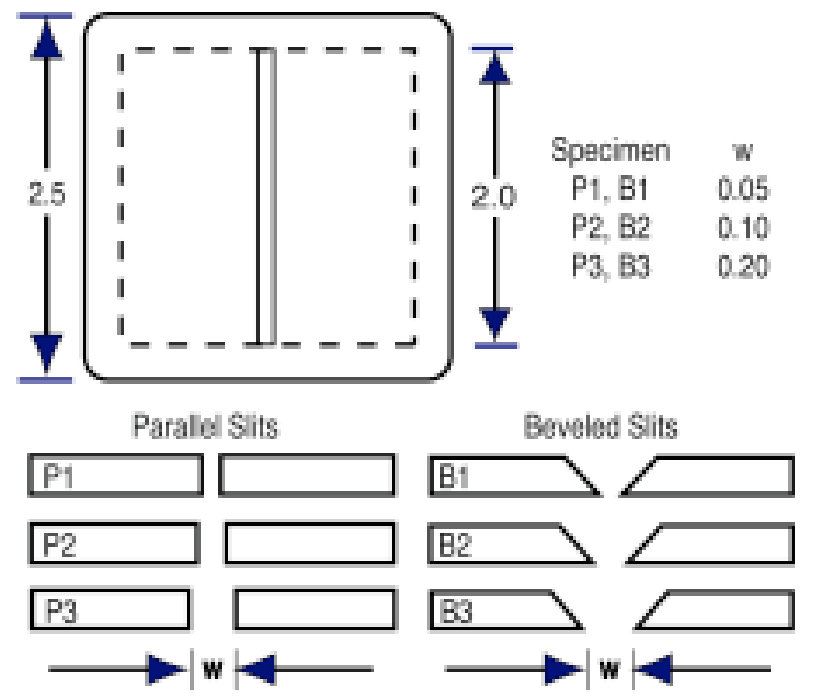

Figure 2. Sketch of slit specimens (dimensions in inches).

\section{Computational Model and Grid Design}

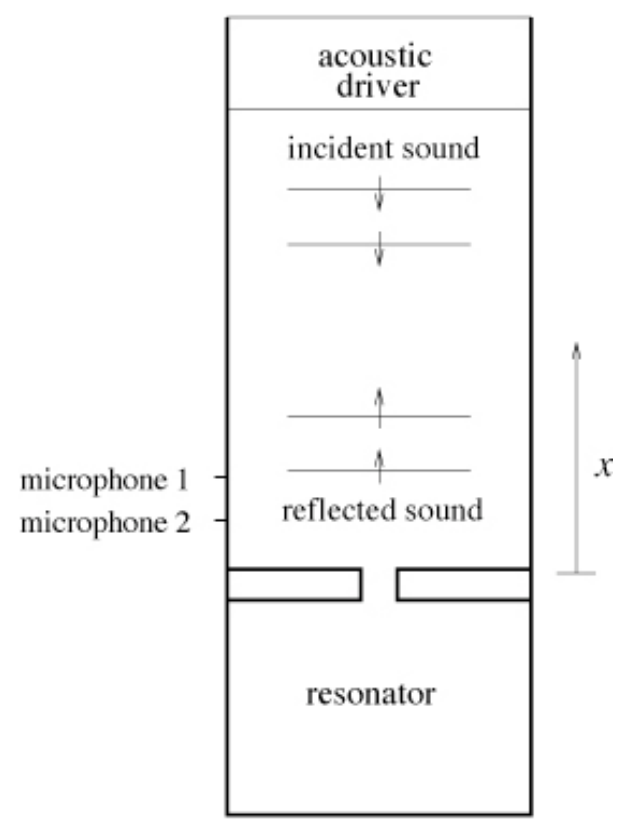

Figure 3. Virtual normal incident impedance tube model used for direct numerical simulation.

An impedance tube is a standard apparatus for measuring the normal incidence of an acoustic liner at a chosen measurement plane. The impedance tube model (virtual impedance tube) for this test consists of a long tube, one end of which is partitioned into a resonator by a plate with a slit as shown in figure 3. Physically, the aspect ratio of the slit is large so that a two-dimensional model is considered to be adequate. At the other end of the tube is an acoustic driver. The acoustic driver generates plane acoustic waves that propagate down the tube until they strike the plate separating the resonator and the rest of the impedance tube. Part of the incident waves is reflected back and part of the waves passes through the slit into the resonator. Two virtual microphones are mounted on walls of the impedance tube. They are used to measure the pressure time history inside the tube. Analysis of the two pressure signals allows the acoustic impedance of the resonator to be determined.

\section{A. Mathematical Model}

The flow and acoustic field inside an impedance tube are governed by the compressible Navier-Stokes equations. However, viscous effects are important only in the region in or near the slit. Away from the slit, the compressibility effect is most important. Thus the governing equations are switched to the Euler equations outside the immediate vicinity of the slit.

The following scales are used to nondimensionalize the flow variables: length scale $=D$ (the thickness of the slit opening), velocity scale $=a_{0}$ (the speed of sound), density scale $=\square_{0}$ (the gas density in the absence of incident sound), and pressure and stress scale $=\square_{0} a_{0}^{2}$. The governing equations are,

$$
\begin{gathered}
\frac{\partial \square}{\partial t}+\square \frac{\partial u_{j}}{\partial x_{j}}+u_{j} \frac{\partial \square}{\partial x_{j}}=0 \\
\frac{\partial u_{i}}{\partial t}+u_{j} \frac{\partial u_{i}}{\partial x_{j}}=\square \frac{1}{\square} \frac{\partial p}{\partial x_{i}}+\frac{1}{\square} \frac{\partial \square_{i j}}{\partial x_{j}} \\
\frac{\partial p}{\partial t}+u_{j} \frac{\partial p}{\partial x_{j}}+\square \frac{\partial u_{j}}{\partial x_{j}}=0 \\
\square_{i j}=\frac{1}{R_{D}}=\frac{\partial u_{i}}{=}+\frac{\partial u_{j}}{\partial x_{i}} \stackrel{=}{=}
\end{gathered}
$$

$R_{D}=D a_{0} / \square$ is the Reynolds number based on the thickness of the slit and // is the kinematic viscosity of the fluid. 


\section{B. Mesh Design}

The impedance tube problem is a multi-scales problem. Near the walls forming the slit, the viscous effect is dominant. Here the oscillatory flow through the slit, induced by the incident sound, gives rise to a Stokes layer. The wave length, $\Pi$, of an oscillatory Stokes layer is, ${ }^{10}$

$$
\square=2 \stackrel{\square}{=\frac{\square \square}{\square}}
$$

where $f$ is the frequency of oscillation. The 7point Dispersion-Relation-Preserving (DRP) scheme ${ }^{11,12}$, which is used in the simulation, is capable of resolving a wave computationally by using 7 to 8 mesh points per wavelength. Thus the mesh size required at the slit is $(\square x)_{\text {Stokes }}=\square / 8=0.8(\square / \square f)^{1 / 2}$.

Away from the slit, the fluid motion is dominated by the compressibility effect. The acoustic wavelength is $a_{0} / f$. The resolution requirement for the DRP scheme is, therefore, equal to $(\square x)_{\text {acoustic }}=a_{0} /(8 f)$. The ratio of the mesh size is,

$$
\frac{\square x_{\text {acoustic }}}{\square x_{\text {Stokes }}}=\frac{0.277 a_{0}}{(\square f)^{1 / 2}} .
$$

Under standard conditions, this ratio is equal to 320 for $6 \mathrm{kHz}$ sound. This is a huge ratio and must be addressed in the grid design.

In designing the computational mesh for the impedance tube problem, the frequency range to be resolved is taken to be 0.5 to $6.0 \mathrm{kHz}$. This covers the maximum frequency range of planned NASA experiments (only results for up to $3.0 \mathrm{kHz}$ are reported in this report). To deal with the large disparity of the spatial mesh size requirement, the multi-size-mesh multi-time-step DRP scheme of Tam and Kurbatskii ${ }^{13}$ is employed. This scheme uses uniform mesh size blocks with mesh size change by a factor of two in adjacent blocks. Figure 4 shows the mesh size design inside the resonator. There are eight mesh blocks. The smallest size mesh, $\square_{1}$, is in the slit region (slit is 0.04 " thick) with $\square x=\square y=\square_{1}=0.05 / 80$ inches. This is small enough to resolve the viscous Stokes layer adjacent to the wall. The largest size mesh is $2^{7} \square_{1}$ or $128 \square_{1}$. This is sufficient to resolve acoustic wave frequencies up to $6 \mathrm{kHz}$. Figure 5 shows the mesh design inside the impedance tube but outside the resonator. Again eight layers of mesh are used. The block with the largest size mesh occupies over $85 \%$ of the computation domain. This drastically reduces the amount of computation needed in the simulations.

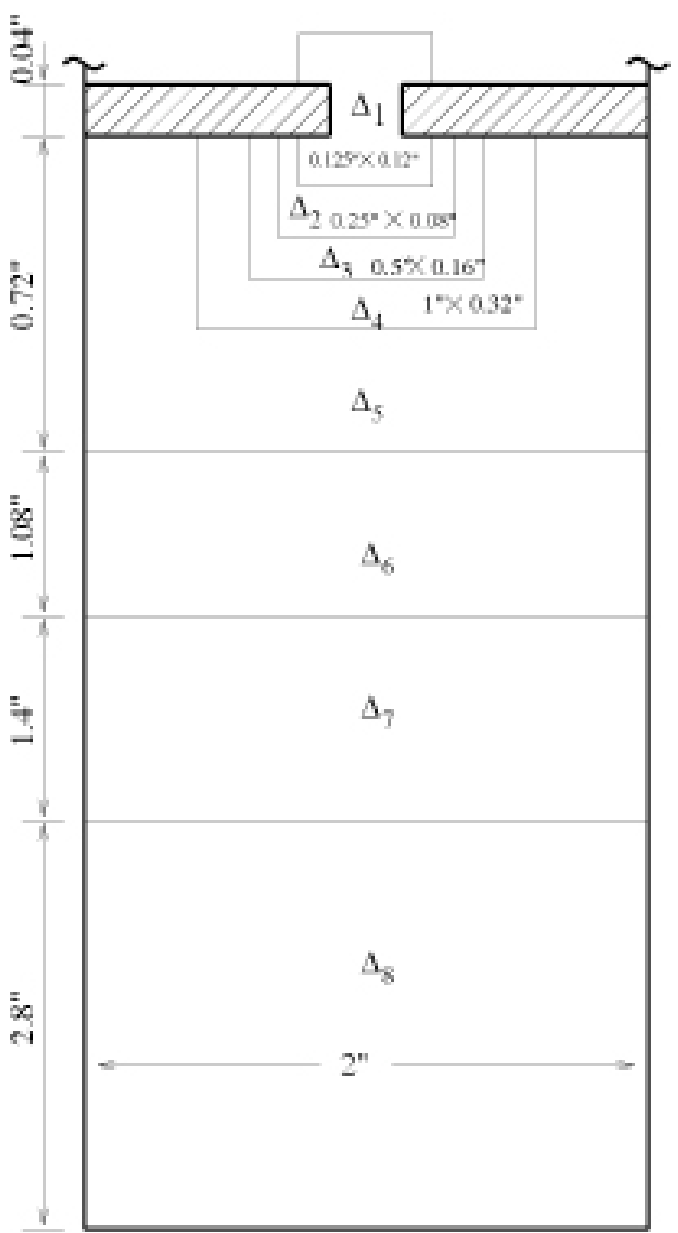

Figure 4. Mesh design inside the resonator.

For simulations involving a 45-degree angle bevel slit, an additional mesh block is inserted at the mouth of the opening as shown in figure 6 . The mesh size is $\square_{1} / 2^{1 / 2}$. Because the walls are now slanted at 45 degrees, an oblique Cartesian mesh is used to maintain a bodyfitted grid. A body-fitted grid is the best for resolving the Stokes layer adjacent to the walls. 


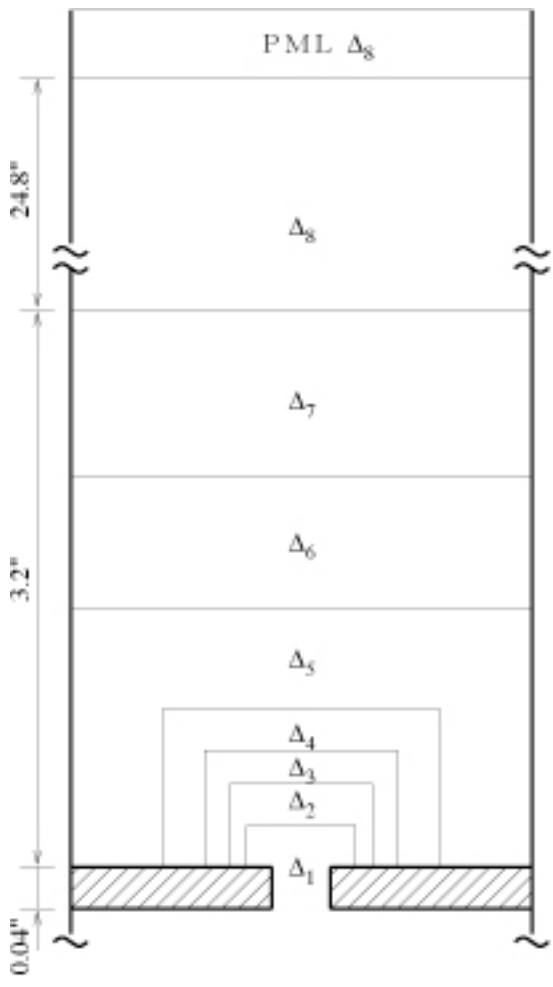

Figure 5. Mesh design inside the impedance tube.

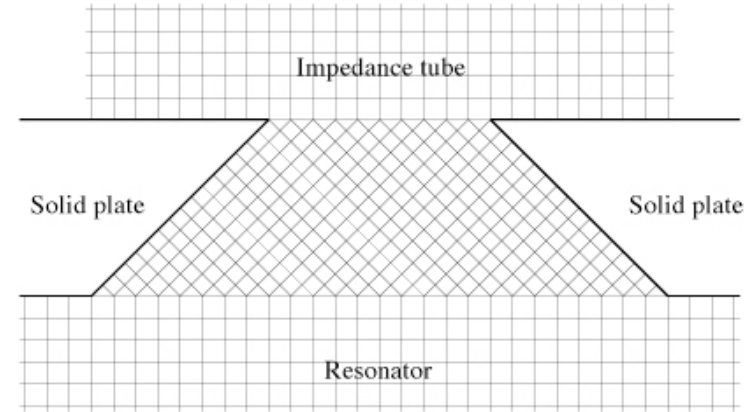

Figure 6. Mesh design for $45^{\circ}$ bevel slit.

\section{Computation Algorithm}

The multi-size-mesh multi-time-step DRP scheme of Tam and Kurbatskii ${ }^{13}$ is used in the simulation. This scheme, designed for multiple-scales problems, is particularly suited for the present computation. One unique feature of this scheme is its multi-timestep marching capability. Most computational fluid dynamics or computational aeroacoustics methods are formulated to use a single time step for all the mesh points. The time step is, therefore, controlled by the stability requirement of the smallest size mesh. This is very inefficient, as a lot of unnecessary computation has to be performed over mesh points with large size mesh. The scheme of Tam and Kurbatskii synchronizes the size of the time step with the spatial mesh size. Thus most of the computations are done over regions with the highest resolution where time scales are shorter. For the present grid design, it is estimated that more than half of the computing effort is spent on the three blocks with the finest meshes.

The DRP scheme uses a central difference quotient for spatial discretization. An optimized four level method is used to advance the solution in time. Since a central difference scheme has no intrinsic numerical damping, artificial selective damping terms ${ }^{14,12}$ are added to all the discretized equations. The artificial selective damping has the property that the damping is concentrated on high wavenumbers or the short waves. The damping rate for waves with low wave number (i.e., the physical solution) is extremely small. Thus artificial selective damping is very effective in removing short spurious waves that may be generated at mesh-size-change interfaces or wall boundaries. On including artificial selective damping, the discretized form of the $y$ momentum equation (equation (2)) according to the DRP scheme is (the discretized forms of the other governing equation are similar),

$$
v_{\ell, m}^{(n+1)}=v_{\ell, m}^{(n)}+\square t \square_{j=0}^{3} b_{j} K_{\ell, m}^{(n \square j)}
$$

$$
\begin{aligned}
& K_{\ell, m}^{(n)}=\square \frac{u_{\ell, m}^{(n)}}{\square x} \square_{j=\square 3}^{3} a_{j} v_{\ell+j, m}^{(n)} \square \frac{v_{\ell, m}^{(n)}}{\square y} \square_{j=\square 3}^{3} a_{j} v_{\ell, m+j}^{(n)} \\
& \square \frac{1}{\square_{\ell, m}^{(n)}} \frac{1}{\square y} \square_{j=\square 3}^{3} a_{j} p_{\ell, m+j}^{(n)} \\
& +\frac{1}{\square_{l . m}^{(n)}} \square_{j=\square 3}^{3} a_{j} \stackrel{\square}{\square \square x}\left(\square_{y x}\right)_{\ell+j, m}^{(n)}+\frac{1}{\square y}\left(\square_{y y}\right)_{\ell, m+j}^{(n)} \underset{E}{[} \\
& \square \frac{1}{R_{\square}} \bigsqcup_{j=\square 3}^{3} d_{j} \stackrel{\square}{\stackrel{\square}{\square} \frac{1}{\square}} v_{\ell+j, m}^{(n)}+\frac{1}{\square y} v_{\ell, m+j}^{(n)} \stackrel{\square}{\longrightarrow}
\end{aligned}
$$

where

$$
\begin{aligned}
& \left.\left(\square_{x y}\right)_{\ell, m}^{(n)}=\frac{1}{R_{D} \square x} \square_{j=\square 3}^{3} a_{j}\right]_{\ell, m+j}^{(n)} \stackrel{\square}{\square} \frac{\square}{\square} \square+v_{\ell+j, m}^{(n)} \stackrel{[}{[}- \\
& \left(\square_{y y}\right)_{\ell, m}^{(n)}=\frac{2}{R_{D} \square y} \square_{j=\square 3}^{3} a_{j} v_{\ell, m+j}^{(n)}
\end{aligned}
$$


$R_{\square}=\left(a_{0} \square\right) / \square_{a}$ is the mesh Reynolds number based on $\square_{a}$ the artificial kinematic viscosity.

The last term of (8) is the artificial selective damping term. By taking the Fourier transform of (8) and (9) (see Ref.[11]) it is easy to find that the ratio of viscous damping to artificial selective damping, in terms of wavenumber $/ /$, is given by

$$
\frac{\text { viscous damping }}{\text { artificial selective damping }} \square \frac{D}{\square x} \frac{R_{\square}}{R_{D}} \frac{(\square \square x)^{2}}{D(\square \square x)}
$$

where $\Pi$ is the stencil wavenumber (see Ref.[11],[12]). It is a function of $\Pi \prod x$. $D(\square \square x)$ is the artificial selective damping function. ${ }^{14}$ For the present problem with a mesh design as given in figures 4 and 5 , it is straightforward to find $R_{D} \square 2.38 \square 10^{4}$. For background damping $R_{\square}$ is given a value of 12.5. Figure 7 shows the ratio of viscous to artificial selective damping. It is clear from this figure that artificial selective damping is important only for short waves. Its inclusion is used to remove spurious numerical waves especially grid-to-grid oscillations. Along mesh-size-change interfaces and wall boundaries, a smaller mesh Reynolds number is used. This increase in artificial selective damping is used to ensure numerical stability by eliminating grid-to-grid oscillations and spurious waves at their origin.

In all the simulations performed, the viscous terms in equation (2) are retained only for the five blocks with the finest meshes. The viscous terms are neglected or the Euler equations are solved in all the other blocks of the computation domain. Accordingly, the noslip boundary conditions are enforced only on walls adjacent to the five blocks with the finest meshes. The inviscid boundary condition is enforced on all other wall surfaces.

At the top of the impedance tube where the acoustic driver is placed in the physical experiment, a Perfectly Matched Layer ${ }^{3}$ (PML) boundary condition is imposed. In this region, all the dependent variables are separated into two parts. The first part is the prescribed incident sound wave. The second part is the wave reflected from the surface of the test specimen. In the PML boundary region, only the reflected waves are computed. They are damped to negligible amplitudes as they propagate through the layer. The PML boundary condition is extremely effective and it does not give rise to any reflected wave.

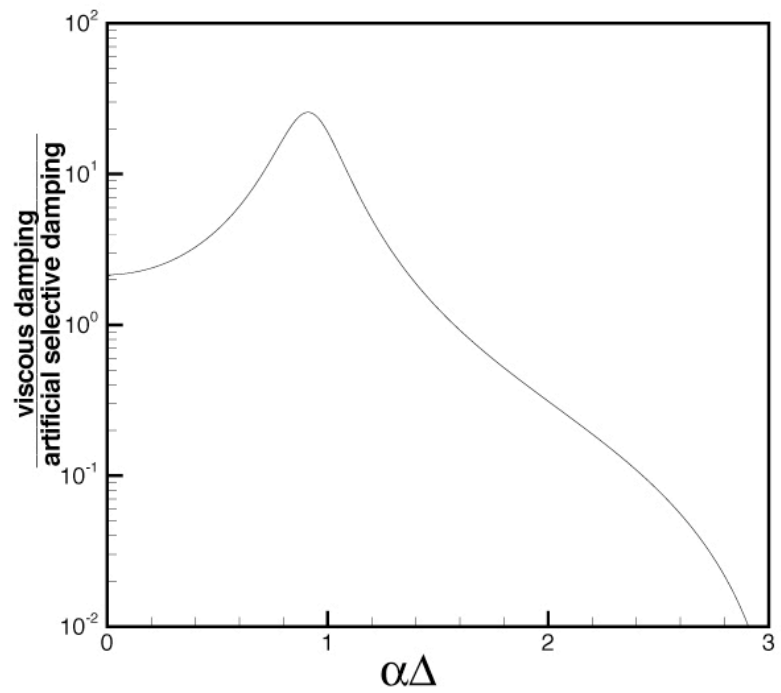

Figure 7. Ratio of viscous damping to artificial selective damping for the first mesh block, $D=0.04$ ", $\square=D / 64, R_{\square}=12.5$, and $R_{D}=2.38 \square 10^{4}$.

\section{Validation of DNS Results and Observations}

To validate DNS results, two series of experiments were carried out at the NASA Langley Research Center. The first series was conducted with a slit with 90-degree corners, while the second series was conducted with a slit with a bevel 45-degree corner. The slit thickness was held constant at 0.04 inches, and slit widths of $0.05,0.1$ and 0.2 inches were used. Six sound frequencies at $0.5,1.0$, 1.5, 2.0, 2.5 and $3.0 \mathrm{kHz}$ were included in the test matrix. Altogether, there were 36 experimental runs. Simultaneously, a parallel effort involving direct numerical simulations of a similar impedance tube was carried out at the Florida State University. By prior agreement, the computed complex reflection factor of the slits at various specified slit widths and incident frequencies and sound pressure levels (SPL) were sent to NASA for comparison with experimental measurements. 
Figures 8 to 13 show the reflection factors obtained by DNS and experiments for the 90degree slit. Figures 8, 10 and 12 give the reflection factor magnitudes. Figures 9,11 and 13 give the corresponding phases. Figures 8 and 9 show the results of the 0.05 -inch slit. Figures 10 and 11 are for the 0.1-inch slit, while figures 12 and 13 are for the 0.2-inch slit. When the DNS results and the experimental measurements were first compared, it was noticed that there were favorable agreements at all frequencies except at $2.5 \mathrm{kHz}$. In an effort to find a possible cause for the discrepancy, the experiments for the 0.05 -inch slit were repeated. It was found that the newly measured data were nearly the same as the original data except for the 2.5 $\mathrm{kHz}$ case. The new measurement at $2.5 \mathrm{kHz}$, unlike the original data, gave a reflection factor that was very close to the DNS results. To ensure that the good agreement with the second series of experiments was not accidental, the experiments were repeated the third time with the slit rotated to a horizontal position instead of a vertical position. As shown in figures 8 and 9, the third set of data was in excellent agreement with the second set of data and the DNS results. This showed that there were three-dimensional effects present in the experimental results. In addition, further experimental investigation at incident SPL's of 120 to $155 \mathrm{~dB}$ subsequently demonstrated that the reflection factor at 2.5 $\mathrm{kHz}$ is extremely sensitive to changes in the incident SPL.

The DNS results shown in figures 10 to 13 for the 0.1 and 0.2 inch width slit with 90-degree corners are also in good agreement with experimental measurements. The exception is again at $2.5 \mathrm{kHz}$. On comparing these two sets of data with those of figures 8 and 9, it becomes clear that the agreement between numerically simulated results and measurements are not as good for the wider slits. Perhaps this is somehow a result of the fact that for a constant acoustic pressure, the acoustic particle velocity is lower for the wider slits (resulting in reduced nonlinearity effects) than for the narrower slit. This issue will be addressed in a future investigation. Also, generally speaking, the phases of the reflection factors are in better agreement than the magnitudes.

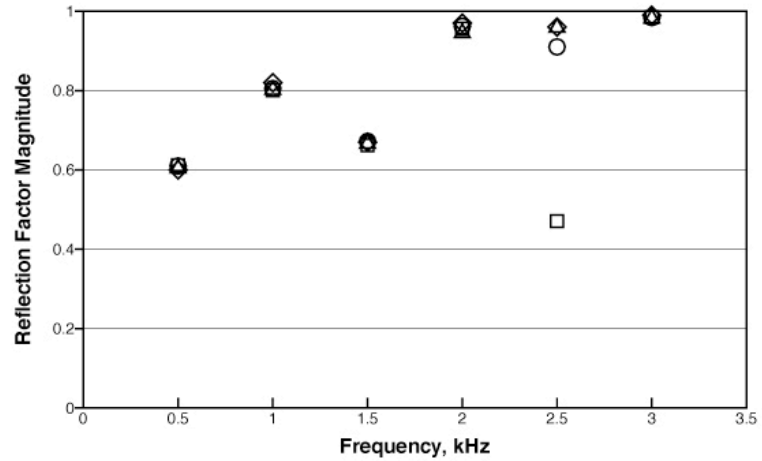

Figure 8. Comparisons between calculated and measured reflection factors of $90^{\circ}$ slit with 0.05 -inch width. $\diamond$ Reflection factor magnitude, calculated; Reflection factor magnitude, measured (vertical slit trial \#1); [ Reflection factor magnitude, measured (vertical slit trial \#2); Reflection factor magnitude, measured (horizontal slit trial \#1). (Time dependence $e^{i \square t}$.)

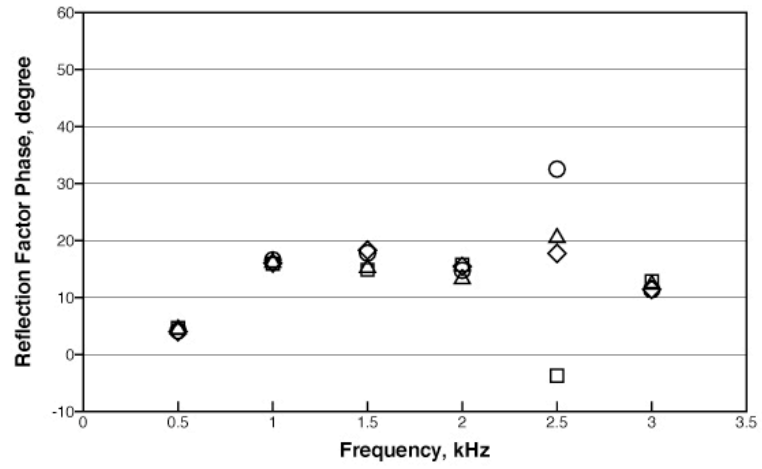

Figure 9. Comparisons between calculated and measured reflection factors of $90^{\circ}$ slit with 0.05 -inch width. $\diamond$ Reflection factor phase, calculated; Reflection factor phase, measured (vertical slit trial \#1); $\triangle$ Reflection factor phase, measured (vertical slit trial \#2); Reflection factor phase, measured (horizontal slit trial \#1).

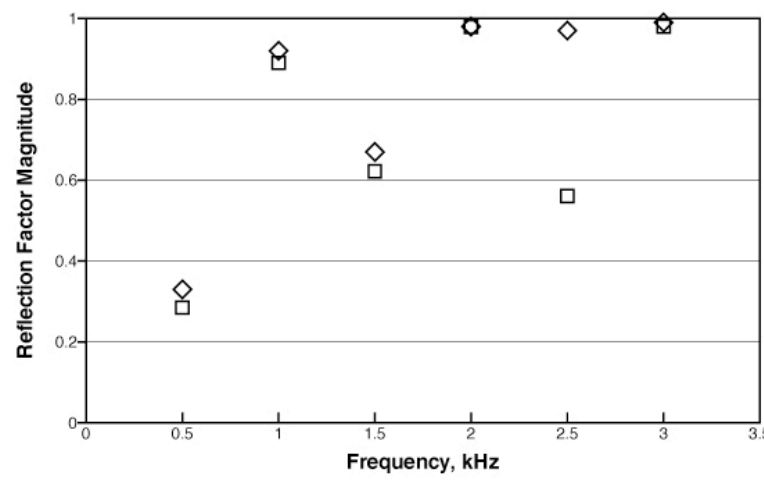

Figure 10. Comparisons between calculated and measured reflection factors of $90^{\circ}$ slit with 0.1-inch width. $\diamond$ Reflection factor magnitude, calculated;

Reflection factor magnitude, measured (vertical slit trial $\# 1)$. 


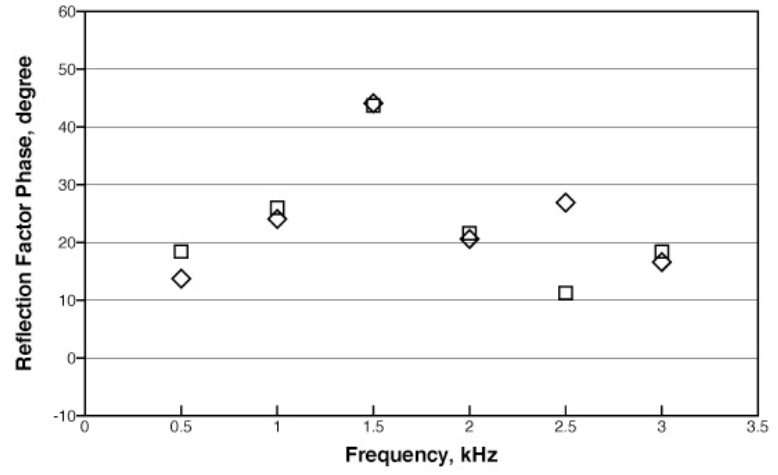

Figure 11. Comparisons between calculated and measured reflection factors of $90^{\circ}$ slit with 0.1 inch width. $\diamond$ Reflection factor phase, calculated; Reflection factor phase, measured (vertical slit trial \#1).

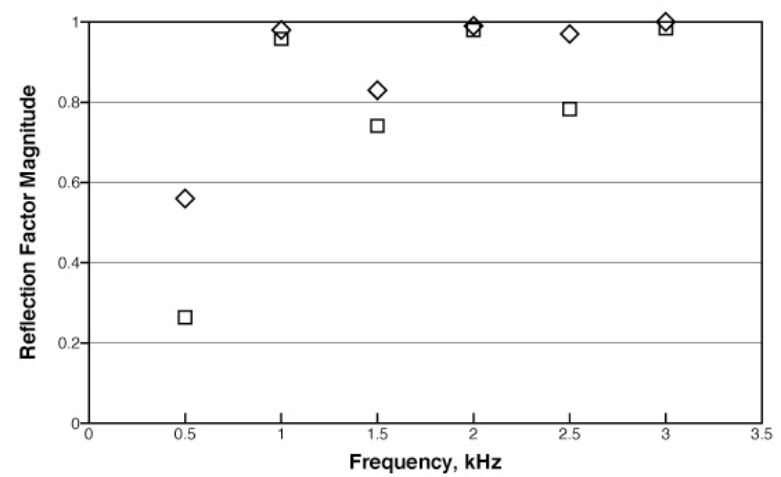

Figure 12. Comparisons between calculated and measured reflection factors of $90^{\circ}$ slit with 0.2 inch width. $\diamond$ Reflection factor magnitude, calculated; Reflection factor magnitude, measured (vertical slit trial \#1).

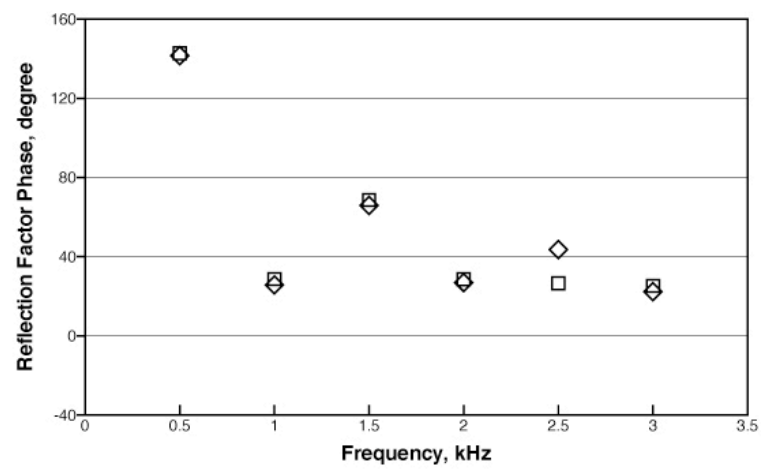

Figure 13. Comparisons between calculated and measured reflection factors of $90^{\circ}$ slit with $0.2-$ inch width. $\diamond$ Reflection factor phase, calculated; Reflection factor phase, measured (vertical slit trial \#1).

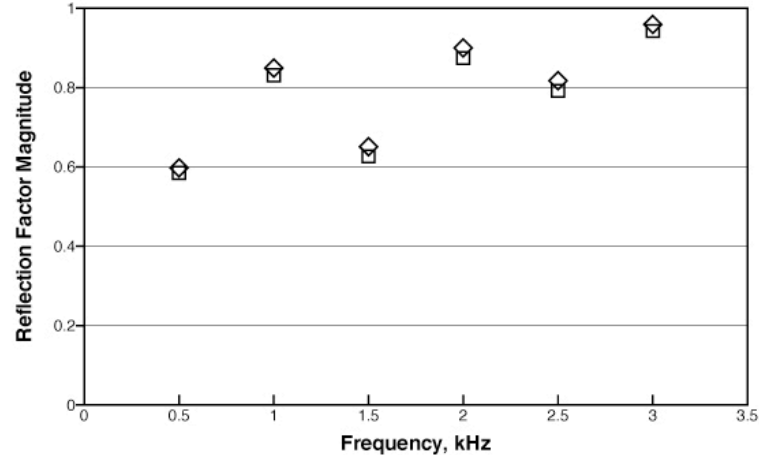

Figure 14. Comparisons between calculated and measured reflection factors of $45^{\circ}$ bevel slit with 0.05 -inch width. $\diamond$ Reflection factor magnitude, calculated; Reflection factor magnitude, measured.

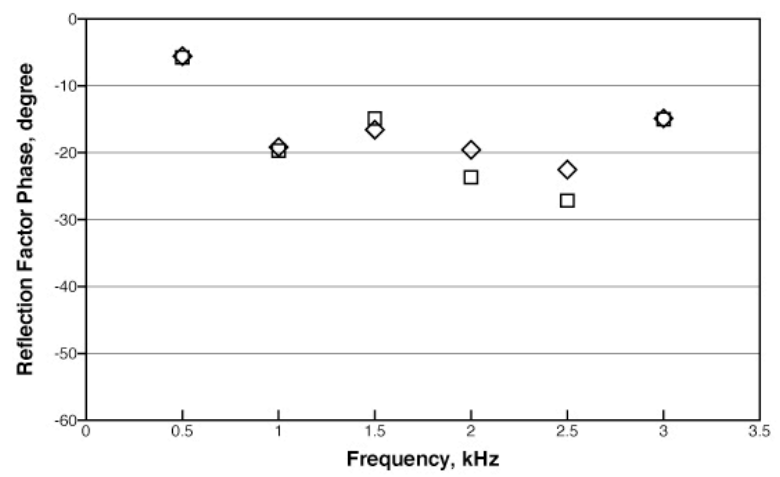

Figure 15. Comparisons between calculated and measured reflection factors of $45^{\circ}$ bevel slit with 0.05 -inch width. $\diamond$ Reflection factor phase, calculated; Reflection factor phase, measured.

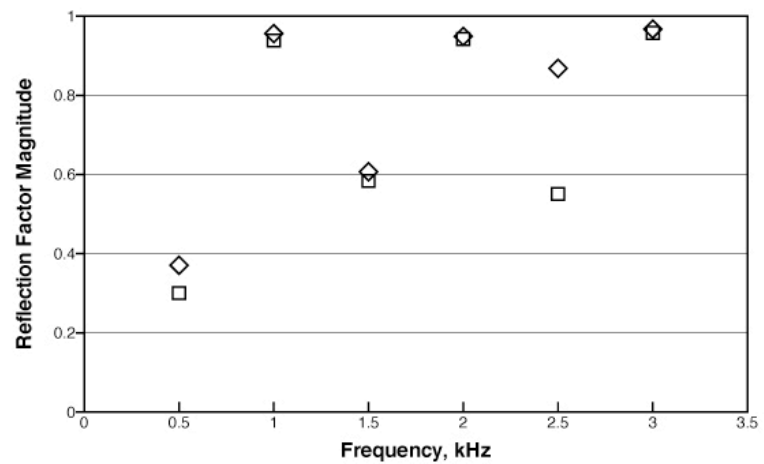

Figure 16. Comparisons between calculated and measured reflection factors of $45^{\circ}$ bevel slit with 0.1-inch width. $\diamond$ Reflection factor magnitude, calculated; Reflection factor magnitude, measured. 


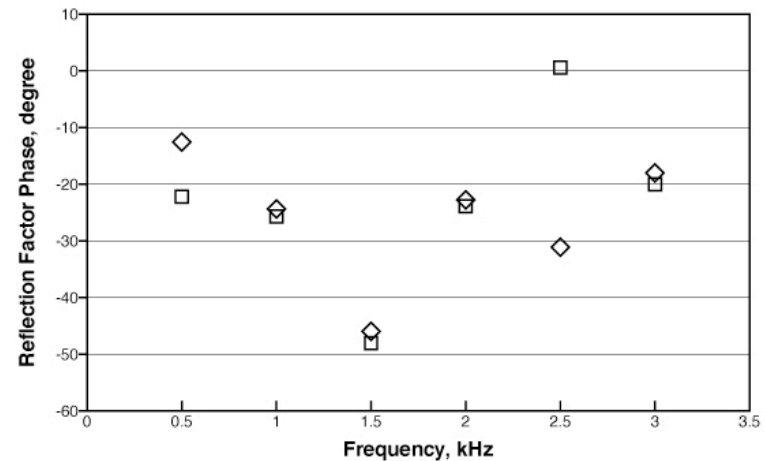

Figure 17. Comparisons between calculated and measured reflection factors of $45^{\circ}$ bevel slit with 0.1 -inch width. $\diamond$ Reflection factor phase, calculated; Reflection factor phase, measured.

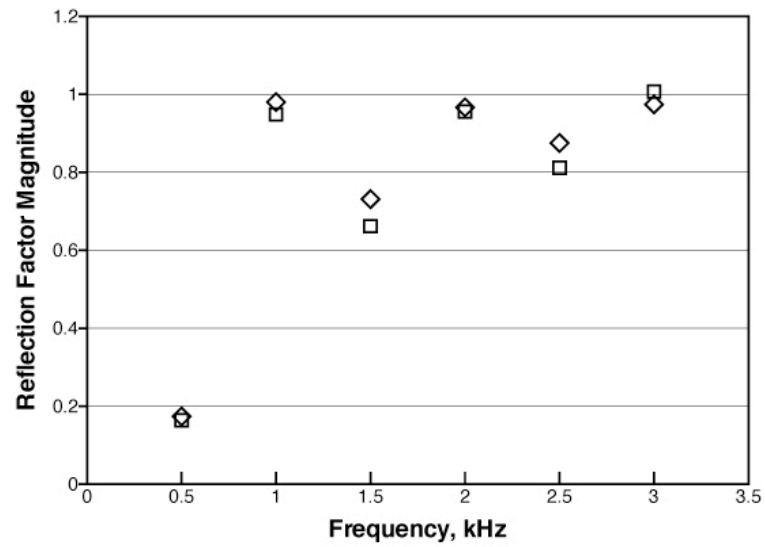

Figure 18. Comparisons between calculated and measured reflection factors of $45^{\circ}$ bevel slit with 0.2-inch width. $\diamond$ Reflection factor magnitude, calculated; Reflection factor magnitude, measured.

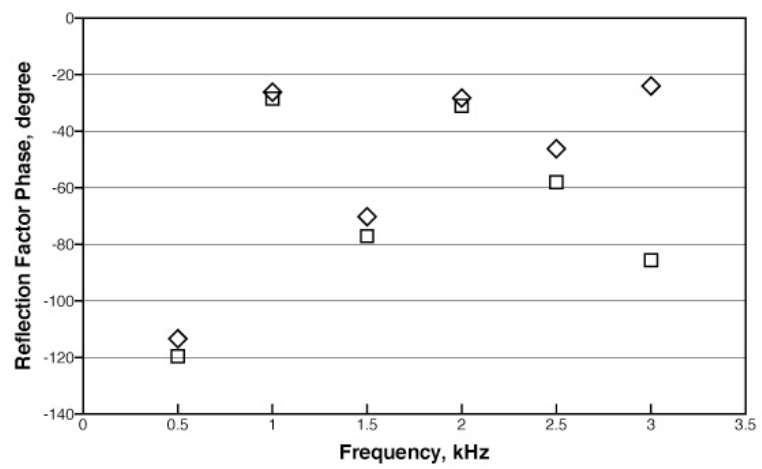

Figure 19. Comparisons between calculated and measured reflection factors of $45^{\circ}$ bevel slit with 0.2 -inch width. $\diamond$ Reflection factor phase, calculated; Reflection factor phase, measured.

The 45-degree beveled slit data is shown in figures 14 to 19 . As can be seen, there is good overall agreement between DNS results and experiments. Again there are significant differences at $2.5 \mathrm{kHz}$. This is particularly true for the 0.1 inch wide slit. At all other frequencies, the agreements are comparable to those of the 90-degree slit.

The main purpose of performing numerical simulations and experimental measurements is to offer a critical test of the validity of DNS results. However, aside from this, the simulations provide a unique opportunity to observe the flow and acoustic field around the slit region. Owing to the smallness of the slit, this is not easy to do experimentally. For this purpose, videos of the simulation data were made. These videos provide the following observations.

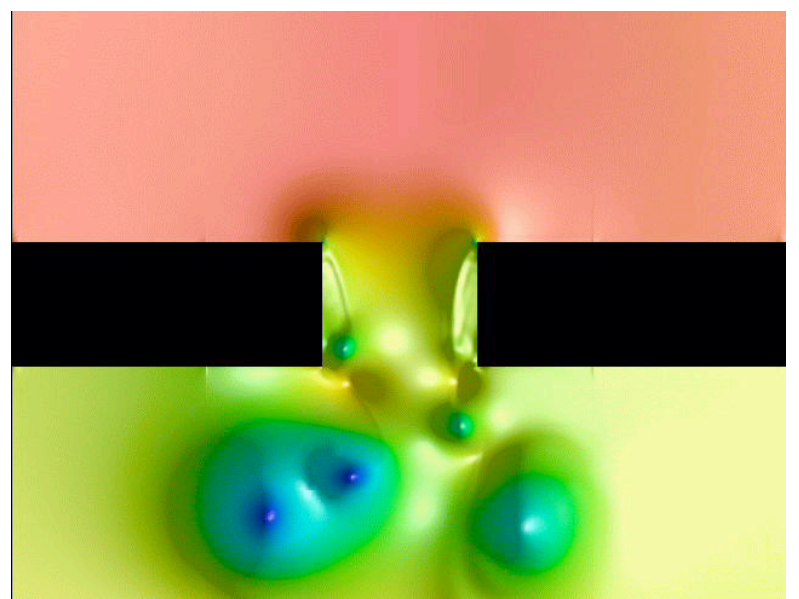

Figure 20. Vortex shedding at a $90^{\circ}$ slit.

For the 90-degree slit, an incident sound pressure level around $140 \mathrm{~dB}$ or higher leads to strong vortex shedding (see figure 20). When the incident sound wave reaches a high pressure outside the resonator, fluid is forced into the cavity through the slit. This causes vortex shedding at the two corners on the cavity side. Usually, a pair of large vortices are first shed. They are followed by random shedding of smaller vortices. Half a cycle later, the pressure of the incident sound outside the resonator is at the lowest level. This causes a reversed outflow from the cavity through the slit. Now vortex shedding takes place at the two corners of the slit on the outside of the resonator. The vortex shedding phenomenon is chaotic. It is coordinated only by the oscillating incident sound pressure level. After the vortices are 
shed, they slowly migrate away from the slit opening by mutual induction. Vortex merging is observed to occur but only as a part of the vortex shedding process. Once vortices become free, they seldom merge to form larger vortices. Instead they simply dissipate slowly by molecular viscosity.

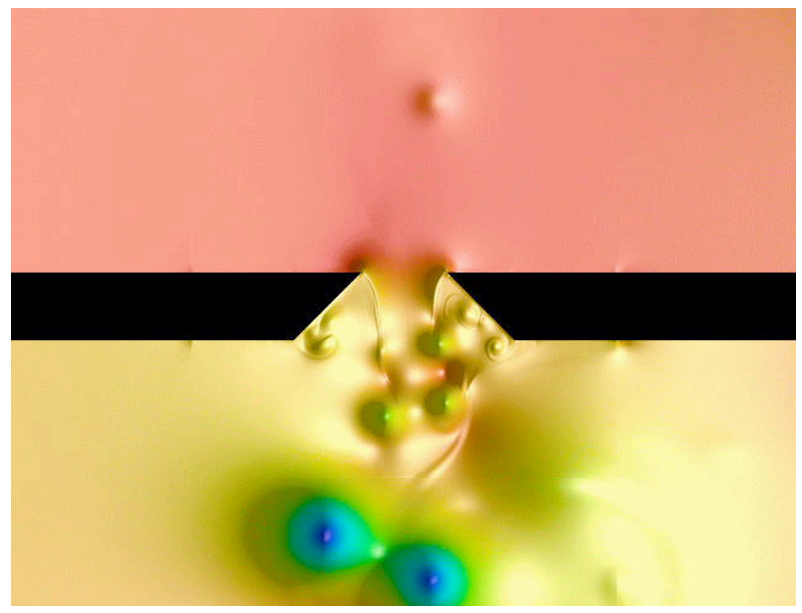

Figure 21. Vortex shedding and the development of thin shear layers and subsequent rolling up into vortices due to Kelvin-Helmholtz instability at a $45^{\circ}$ bevel slit.

For the 45-degree beveled slit, under the same incident sound pressure level and frequency, the vortex shedding process is slightly different. Now there are two sharp 45-degree edges on the outside surface of the resonator and two blunt 135-degree corners on the inside surface. For this slit geometry, most vortex shedding occurs at the sharp 45-degree edge, although some sheddings occur at the blunt 135-degree corners. Regardless of whether the fluid is leaving or entering the resonator by way of the slit, two large vortices are shed from the sharp 45-degree corners of the bevel slit at the beginning of a flow cycle (see figure 21). The two large vortices are usually followed by two thin shear layers; one on each side of the slit. The thin shear layer is unstable to KelvinHelmholtz instability. The instability grows very quickly, causing the thin shear layer to roll up into a string of tiny vortices. Thus, associated with every flow reversal in the slit region is the shedding of two large vortices from the sharp edge of the slit. They are followed by a string of small vortices. Now if the incident sound pressure level is gradually reduced, there is a sound pressure level at which vortex shedding ceases to occur for the slit with 90-degree corners. However, at the same sound pressure level and frequency, vortex shedding continues at the beveled slit. Vortex shedding stops only when the sound pressure level is further reduced.

\section{Broadband Incident Sound Waves}

Inside a jet engine, in addition to tones generated directly by the fan and by the interaction of the wake flow of the fan and the stator, there are high levels of broadband noise. By improving the design of the fan and by increasing the distance between fan and stator, the tone intensity can be drastically reduced. Consequently, acoustic liner suppression of broadband sound is increasingly important. In this section, we will first discuss how to generate broadband incident sound waves computationally. We will then examine computational error incurred in processing simulated broadband data when the total simulation time is relatively short. Comparisons between DNS results and experiments will be reported.

\section{A. Simulation of Broadband Incident Sound}

In this investigation, a discrete frequency model is used to simulate broadband incident sound waves with a prescribed noise spectrum. The formulation of the discrete frequency model is presented below. It will be assumed that the broadband noise is stationary random.

Let the given broadband spectrum of the sound waves be as shown in figure 22. For convenience, the spectrum is assumed to have a finite bandwidth with a minimum frequency of $f_{L}$ and a maximum frequency of $f_{H}$. In other words, the bandwidth $w$ is equal to $\left(f_{H} \square f_{L}\right)$. The discrete frequency model reproduces a given broadband spectrum computationally by summing over cosine functions, each representing a small band of frequencies. To incorporate phase randomness in the model, the chosen frequencies of the cosine functions are 
incommensurate. Suppose the spectrum is divided into $N$ bands. To produce a sequence of incommensurate frequencies, the bandwidths are taken to increase as a geometric series with a ratio $r(r>1.0$, not an integer) so that if $w_{j}$ denotes the width of the $j^{\text {th }}$ band, then

$$
w_{j}=r w_{j \square 1} .
$$

The total bandwidth is

$$
\begin{aligned}
f_{H} \square f_{L} & =\square_{j=1}^{N} w_{j}=w_{1}\left(1+r+r^{2}+\ldots+r^{(N \square 1)}\right) \\
& =w_{1} \square r r_{\square}^{N} \square 1 \square \\
\text { Thus } \quad & w_{1}=\left(f_{H} \square f_{L}\right) \frac{(r \square 1)}{\left(r^{N} \square 1\right)} .
\end{aligned}
$$

Once $w_{1}$ is found, the width of the $j^{\text {th }}$ band may be calculated by

$$
w_{j}=w_{1} r^{j \square 1} .
$$

The $j^{\text {th }}$ band will be represented by the frequency located at the center of the band. The frequency is

$$
f_{j}=f_{L}+w_{1} \frac{\left(r^{j}+r^{j \square 1} \square 2\right)}{2(r \square 1)} .
$$

By means of (12) and (13), the function representing the pressure of the broadband incident sound waves may be written as,

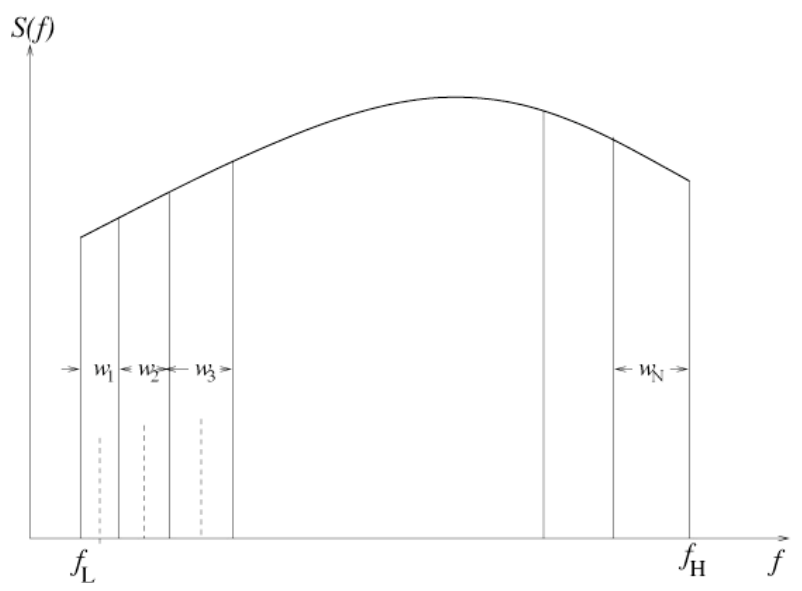

Figure 22. A discrete frequency model of a broadband spectrum.

$$
p(t)=\bigsqcup_{j=1}^{N}\left(2 w_{j} S_{j}\right)^{\frac{1}{2}} \cos \left(2 \square f_{j} t+\square_{j}\right)
$$

where $S_{j}$ is the spectrum level at $f_{j}$ (see figure 22) and $\square_{j}\left(0 \square \square_{j}<2 \square\right)$ is a randomly assigned number.

Figure 23 shows a time history of a broadband spectrum generated by formula (14). Here the spectrum is assumed to be white noise from $f_{L}=0.5 \mathrm{kHz}$ to $f_{L}=3.0$ $\mathrm{kHz}$. The sound pressure level is $152 \mathrm{~dB}$. The spectrum consists of 100 bands with $r=1.01$. As can be seen easily, the time history looks very similar to that of a truly random spectrum.

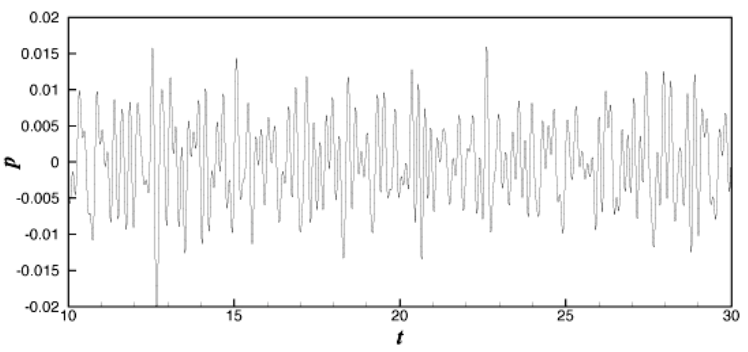

Figure 23. Time history of a simulated broadband spectrum, $f_{L}=0.5 \mathrm{kHz}, f_{H}=3.0$ $\mathrm{kHz}, N=100, r=1.01, \mathrm{SPL}=152 \mathrm{~dB}$.

\section{B. Impedance Computation and Error Estimate}

One of the severe limitations of DNS at the present time is that it takes a large amount of wall clock time to generate a relatively short data segment. This is because a large number of mesh points are needed for accurate numerical simulation of the flow and acoustic fields. As a result, computer time per time step of a simulation is long.

To measure acoustic impedance, a popular way is to use the two-microphone method. 8,9 However, it must be pointed out that the short data sampling time available in data processing imposes a severe constraint on the accuracy of this method when used in DNS computations. In this section, formulas are developed to calculate the acoustic impedance of a slit resonator by the twomicrophone method for broadband incident sound waves. A formula for estimating the 
relative error due to short data sampling time will also be established. This formula was used to select the frequencies of the discrete frequency model (to minimize relative error) in the simulations to be reported below.

Let $x$ be the distance measured along the length of the impedance tube from the face of the slit as shown in figure 3 . We will denote the locations of the two microphones as $x_{1}$ and $x_{2}$. Let $k_{m}$ and $\square_{m}(m=1,2, \ldots, N)$ be the acoustic wavenumbers and angular frequencies of the incident sound waves. Since there is no flow in the tube, the reflected waves will have the same wavenumber. The pressure in the impedance tube may, therefore, be written in the form,

$$
\begin{aligned}
p(x, t)=\square_{m=1}^{N} & \left\{A_{m} \cos \left(k_{m} x+\square_{m} t+\square_{m}\right)\right. \\
& \left.+B_{m} \cos \left(k_{m} x \square \square_{m} t+\square_{m}\right)\right\}
\end{aligned}
$$

where $A_{m}$ and $B_{m}$ are the unknown incident and reflected wave amplitudes. $\square_{m}$ and $\square_{m}$ are the unknown phases. At the two microphones 1 and 2, we have

$$
\begin{gathered}
p_{1}(t)=p\left(x_{1}, t\right) \\
p_{2}(t)=p\left(x_{2}, t\right) .
\end{gathered}
$$

Let $T$ denote the computed data length in time. An overbar will be used to denote an average over time interval $T$. The following correlations can be easily established.

$$
\begin{gathered}
\lim _{T \square} \overline{p_{1}(t) p_{1}(t+\square)} \equiv \lim _{T \square} \frac{1}{T} \prod_{0}^{T} p_{1}(t) p_{1}(t+\square) d t \\
=\square_{m=1}^{N}\left\{\frac{1}{2}\left(A_{m}^{2}+B_{m}^{2}\right)\right. \\
\left.+A_{m} B_{m} \cos \left(2 k_{m} x_{1}+\square_{m}+\square_{m}\right)\right\} \\
\cdot \cos \left(\square_{m} \square\right) \\
\lim _{T \square} \frac{p_{2}(t) p_{2}(t+\square)}{\square}\left\{\square _ { m = 1 } ^ { N } \left\{\frac{1}{2}\left(A_{m}^{2}+B_{m}^{2}\right)\right.\right. \\
\left.+A_{m} B_{m} \cos \left(2 k_{m} x_{2}+\square_{m}+\square_{m}\right)\right\} \\
\cdot \cos \left(\square_{m} \square\right)
\end{gathered}
$$

$$
\begin{aligned}
\lim _{T \square} & \overline{p_{1}(t) p_{2}(t+\square)}=\prod_{m=1}^{N}\left\{\frac{1}{2}\left(A_{m}^{2}+B_{m}^{2}\right)\right. \\
& \cdot \cos \left[k_{m}\left(x_{1} \square x_{2}\right)\right] \cos \left(\square_{m} \square\right) \\
& +\frac{1}{2}\left(A_{m}^{2} \square B_{m}^{2}\right) \sin \left[k_{m}\left(x_{1} \square x_{2}\right)\right] \\
& \cdot \sin \left(\square_{m} \square\right)+A_{m} B_{m} \\
& \cdot \cos \left[k_{m}\left(x_{1}+x_{2}\right)+\square_{m}+\square_{m}\right] \\
& \left.\cdot \cos \left(\square_{m} \square\right)\right\}
\end{aligned}
$$

The cosine decomposition of the above correlation function leads to,

$$
\begin{aligned}
& \lim _{T \square} \overline{p_{1}^{2}\left(\square_{m}\right)} \equiv \lim _{T \square} \frac{2}{T} \square_{0}^{T} \overline{p_{1}(t) p_{1}(t+\square)} \\
& \cdot \cos \left(\square_{m} \square\right) d \square \\
& =\frac{1}{2}\left(A_{m}^{2}+B_{m}^{2}\right)+A_{m} B_{m} \\
& \cdot \cos \left[2 k_{m} x_{1}+\square_{m}+\square_{m}\right] \\
& \lim _{T \square} \overline{p_{2}^{2}\left(\square_{m}\right)}=\frac{1}{2}\left(A_{m}^{2}+B_{m}^{2}\right) \\
& +A_{m} B_{m} \cos \left[2 k_{m} x_{2}+\square_{m}+\square_{m}\right] \\
& \lim _{T \square} \overline{p_{1} p_{2}\left(\square_{m}\right)}=\lim _{T \square} \frac{2}{T} \prod_{0}^{T} \overline{p_{1}(t) p_{2}(t+\square)} \\
& \cdot \cos \left(\square_{m} \square\right) d \square \\
& =\frac{1}{2}\left(A_{m}^{2}+B_{m}^{2}\right) \\
& \cdot \cos \left[k_{m}\left(x_{1} \square x_{2}\right)\right]+A_{m} B_{m} \\
& \cdot \cos \left[k_{m}\left(x_{1}+x_{2}\right)+\square_{m}+\square_{m}\right]
\end{aligned}
$$

The quantities on the left side of equations (21), (22) and (23) can be calculated from the numerical simulation data. With three equations, the unknown wave amplitudes and phases may be found by recasting these three equations in the following form,

$$
\begin{aligned}
& \tan \left[k_{m}\left(x_{1}+x_{2}\right)+\square_{m}+\square_{m}\right] \\
& =\frac{\left[\overline{p_{2}^{2}\left(\square_{m}\right)} \overline{p_{1}^{2}\left(\square_{m}\right)}\right] \sin \left[k_{m}\left(x_{1} \square x_{2}\right)\right]}{2 p_{1} p_{2}\left(\square_{m}\right)}\left[\overline{p_{1}^{2}\left(\square_{m}\right)} \overline{\left.p_{2}^{2}\left(\square_{m}\right)\right] \cos \left[k_{m}\left(x_{1} \square x_{2}\right)\right]}\right. \\
& A_{m} B_{m}=\frac{\left[\overline{p_{2}^{2}\left(\square_{m}\right)} \square \overline{\left.p_{1}^{2}\left(\square_{m}\right)\right]}\right.}{2 \sin \left[k_{m}\left(x_{1}+x_{2}\right)+\square_{m}+\square_{m}\right] \sin \left[k_{m}\left(x_{1} \square x_{2}\right)\right]}
\end{aligned}
$$




$$
\begin{aligned}
A_{m}^{2}+B_{m}^{2}= & \overline{p_{1}^{2}\left(\square_{m}\right)}+\overline{p_{2}^{2}\left(\square_{m}\right)} \square 2 A_{m} B_{m} \\
& \cdot \cos \left[k_{m}\left(x_{1}+x_{2}\right)+\square_{m}+\square_{m}\right] \\
& \cdot \cos \left[k_{m}\left(x_{1} \square x_{2}\right)\right]
\end{aligned}
$$

The right side of (24) is known from simulation data. (24) can be used to determine the unknown phase $\left(\square_{m}+\square_{m}\right)$. Once $\left(\square_{m}+\square_{m}\right)$ is found, $A_{m} B_{m}$ may be calculated by (25) and $\left(A_{m}^{2}+B_{m}^{2}\right)$ may be calculated by (26). With $A_{m} B_{m}$ and $\left(A_{m}^{2}+B_{m}^{2}\right)$ determined, $\left(A_{m}+B_{m}\right)^{2}$ and $\left(A_{m} \square B_{m}\right)^{2}$ can be computed by simple addition and subtraction so that $A_{m}$ and $B_{m}$ can be calculated. In other words, the incident and reflected wave amplitudes are found. On taking the time factor to be of the form $e^{i \square_{m} t}$, it is easy to find that the acoustic impedance, $z_{m}$, and absorption coefficient, $\square_{m}$, at frequency $f_{m}=\square_{m} /(2 \square)$ are given by the formulae below,

$$
\begin{gathered}
z_{m}=\frac{A_{m}+B_{m} e^{\square i\left(\square_{m}+\square_{m}\right)}}{A_{m} \square B_{m} e^{\square i\left(\square_{m}+\square_{m}\right)}} \\
\square_{m}=1 \square B_{m}
\end{gathered}
$$

In deriving formulae (24) to (28), it is explicitly assumed that the data sampling time $T$ is very long. Unfortunately, numerical simulation usually does not provide long sampling data. For the purpose of making an estimate of the error incurred in using a finite $T$, one may use the largest term neglected in replacing $T$ by $T \Pi$ in equations (18) to (20). It is straightforward to find the relative error is,

$$
\text { Relative error } \square \frac{A_{j}}{A_{m}} \frac{1}{T\left(\square_{m} \square \square_{j}\right)}
$$

The relative error is largest when $j=(m \square 1)$. On considering that the wave amplitude does not change greatly between adjacent frequency band; i.e., $A_{j} \square A_{j \square 1}$, the relative error becomes

$$
\text { Relative error } \square \frac{1}{T\left(\square_{m} \square \square_{m \square 1}\right)}
$$

To achieve a relative error of less than $/ \%$, the simulation time, $T$, must be longer than $100 /\left[\square\left(\square_{m} \square \square_{m \square 1}\right)\right], m=1,2, \ldots, N$.

\section{Comparisons with Experiments}

Experiments with white noise input were performed at the NASA Langley Research Center using both the slit with 90-degree corners and the 45-degree bevel slit. This set of data allows us to test the validity of DNS when the incident sound is broadband. In the experiment, the slit width was set at 0.05 inch. Two incident overall sound pressure levels (integrated over frequency range of 0.5 to $3.0 \mathrm{kHz}$ ) were used. One was at $120 \mathrm{~dB}$ and the other at $140 \mathrm{~dB}$. The measured acoustic impedances of the slit resonators using the two-microphone method are shown in figures 24 to 27. Numerical simulations of the virtual impedance tube under conditions closely approximating those of the experiments were carried out at the Florida State University. In the simulations, the broadband incident sound was produced according to the discrete frequency model. The following model parameters were used.

$$
\begin{gathered}
f_{L}=0.5 \mathrm{kHz}, f_{H}=3.0 \mathrm{kHz}, \\
f_{L}=r=1.01, N=22
\end{gathered}
$$

To keep the relative error due to short simulation time to no more than $1 \%$, the simulations were continued for 58 periods of the lowest frequency of the spectrum (period $=0.0018$ sec.). This choice was based on equation (30). The calculated resistances and reactances over the entire frequency band are plotted in figures 24 to 27 . On comparing the calculated and measured results, it is evident that there is good overall agreement, although the comparisons for low frequencies with the 45-degree beveled slit are poor. The reactances for both the 90-degree slit and the 45-degree beveled slit agree well up to 2.5 $\mathrm{kHz}$. The experimental data indicates an antiresonance at approximately $2.6-2.7 \mathrm{kHz}$. The simulations, however, do not encounter this anti-resonance. The reason for this discrepancy is not known. However, it should be noted that measurement difficulties in the frequency region near anti-resonance are not uncommon. 
The average levels of resistance computed in each case are in reasonably good agreement with experiment. Apparently due to the fact that only 22 frequency bands are used to represent the broadband spectrum, the anti-resonance frequencies at approximately 1.1, 2.2 and 2.6 $\mathrm{kHz}$ are not reproduced well in the simulations. This is because the half-width of the resonances are too narrow to be adequately captured by the

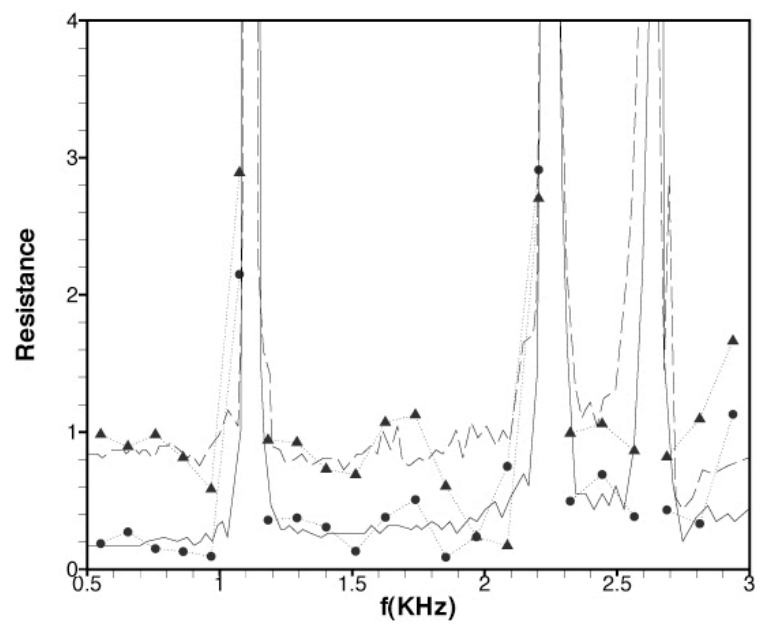

Figure 24. Comparison between calculated and measured resistance of a $90^{\circ}$ slit with 0.05 -inch width. Broadband incident sound, $-120 \mathrm{~dB}$ experiment, $\cdots \square \cdots 120 \mathrm{~dB}$ calculation, - - $140 \mathrm{~dB}$ experiment, $\cdots \square \cdot \cdots 140 \mathrm{~dB}$ calculation.

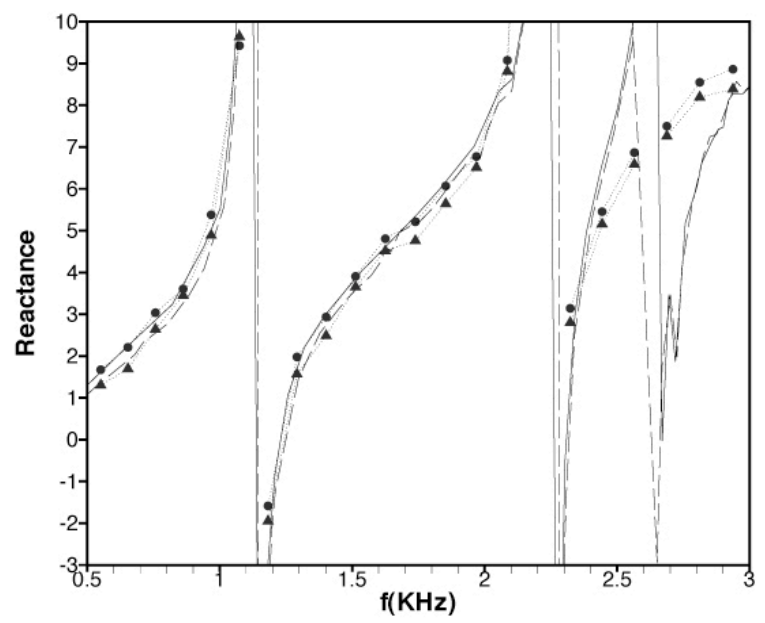

Figure 25. Comparison between calculated and measured reactance of a $90^{\circ}$ slit with 0.05 -inch width. Broadband incident sound, $-120 \mathrm{~dB}$ experiment, $\cdots \square \cdots \quad 120 \mathrm{~dB}$ calculation, - -140dB experiment, ... $\cdots$ 140dB calculation. coarse frequency grid employed in the broadband model of the simulation. It is our belief that had a larger number of frequency bands been used, a better agreement would be obtained. However, to maintain the same low relative error, the simulation time would have to be significantly lengthened. This was not considered feasible and was not implemented.

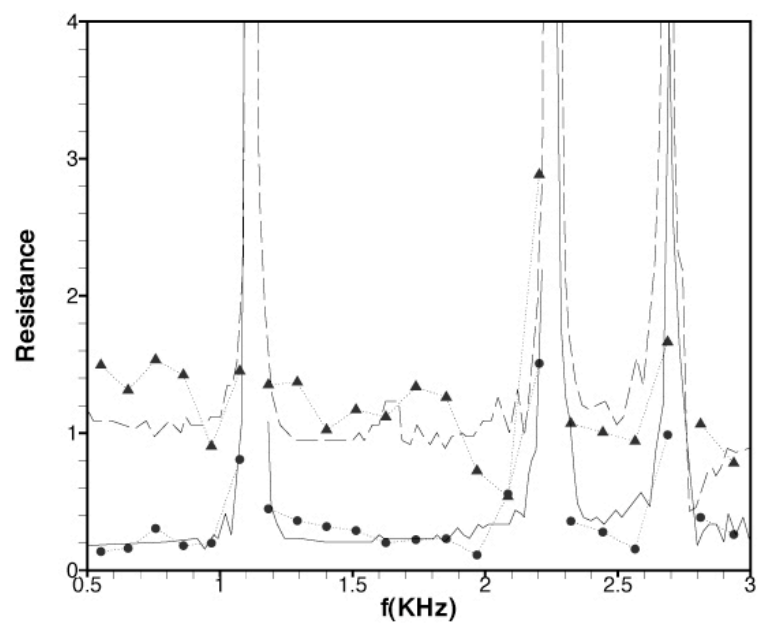

Figure 26. Comparison between calculated and measured resistance of a $45^{\circ}$ bevel slit with 0.05 inch width. Broadband incident sound, $-120 \mathrm{~dB}$ experiment, $\cdots \square \cdots 120 \mathrm{~dB}$ calculation, $-\cdots$ $140 \mathrm{~dB}$ experiment, $\cdots \square \cdot \cdots 140 \mathrm{~dB}$ calculation.

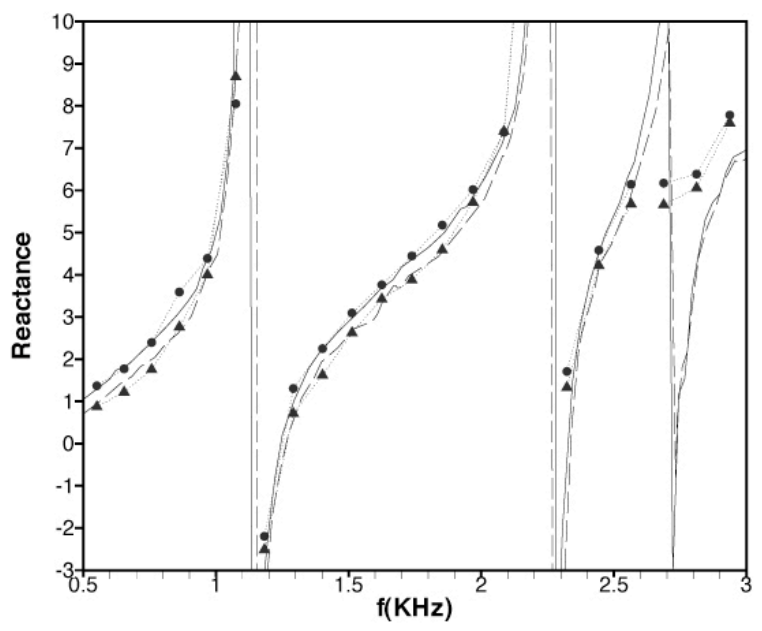

Figure 27. Comparison between calculated and measured reactance of a $45^{\circ}$ bevel slit with 0.05 inch width. Broadband incident sound, $-120 \mathrm{~dB}$ experiment, ${ }^{\cdots}{ }^{\circ} \cdots 120 \mathrm{~dB}$ calculation, - - $140 \mathrm{~dB}$ experiment, $\cdots \square \cdot \cdots 140 \mathrm{~dB}$ calculation. 
To further validate numerical results, the NASA Langley Research Center experiments were repeated using a pseudo-random noise input. As mentioned in Section 2.2, the incident sound consists of thirteen discrete frequencies with prescribed amplitudes and phases. Because the vortex shedding phenomenon is nonlinear, it is expected that the effect of different frequencies would be coupled. This coupling results from achieving sufficient acoustic particle velocity through the slit to trigger this nonlinearity. Thus, these experiments would provide a test of whether numerical simulation can duplicate possible nonlinear coupling of a large number of discrete frequencies.

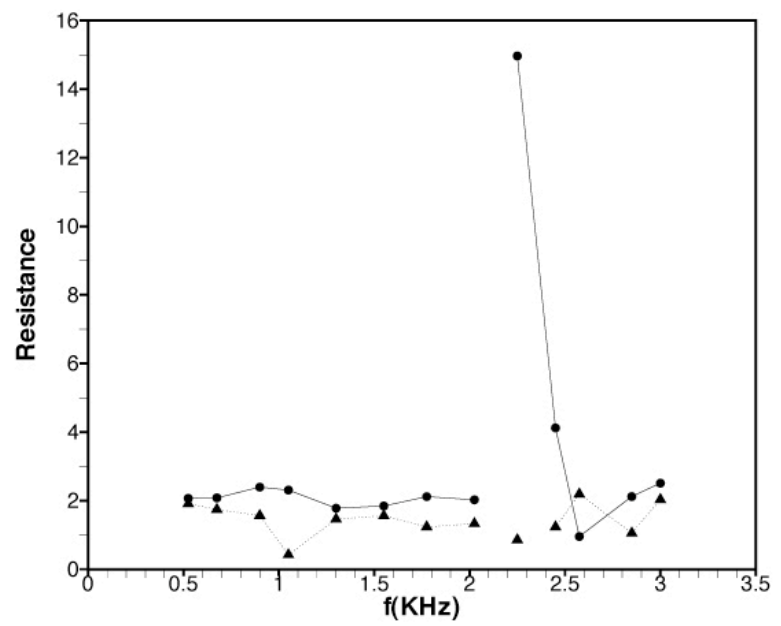

Figure 28. Comparison between calculated and measured normalized resistance for the $90^{\circ}$ slit with 0.05-inch width under pseudo-random acoustic excitation. $\square-$ Experiment, $\cdots \square . .$. DNS.

Figures 28 and 29 show comparisons of the measured and computed normalized acoustic impedance of the 90-degree slit. There appears to be an anti-resonance around 2.2 to $2.3 \mathrm{kHz}$. Because of the wide spacing of the frequency bands, this anti-resonance is not well resolved by both experiment and DNS. At an anti-resonance, the reactance has a jump discontinuity. The structure of the jump discontinuity is shown in figures 25 and 27. On one side of the jump discontinuity, the reactance is positive. On the other side, the reactance is negative. In figure 29 , it is likely that the jump discontinuity is at $2.25 \mathrm{kHz}$. Due to minor differences between experiment and numerical simulation, the experimental data point is on one side of the jump discontinuity while the DNS data point is on the other side. Outside the anti-resonance frequency region, there is general agreement between experiment and DNS results. Figures 30 and 31 show similar comparisons for the 45-degree beveled slit. Other than a couple of isolated points, there is good agreement between measurements and computed results. The agreement is especially good for the normalized reactance.

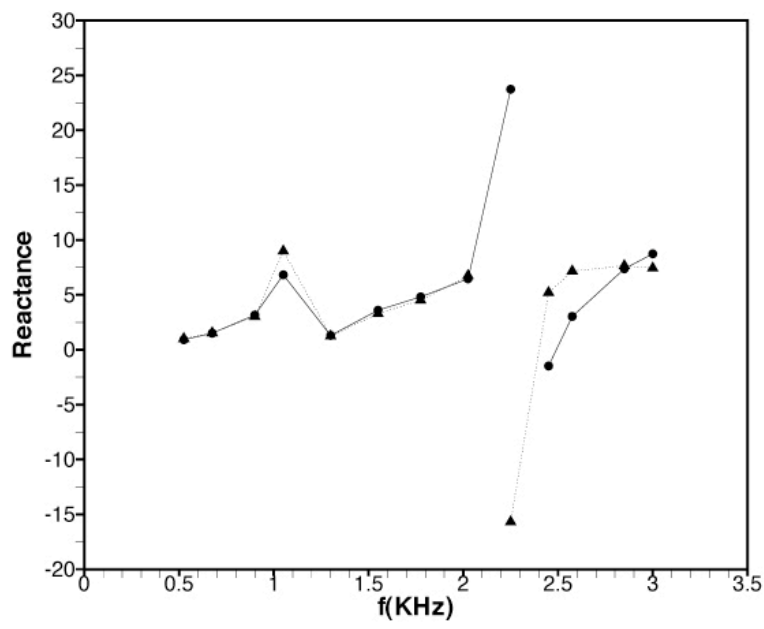

Figure 29. Comparison between calculated and measured normalized reactance for the $90^{\circ}$ slit with 0.05-inch width under pseudo-random acoustic excitation. $\square-$ Experiment, .... ... DNS.

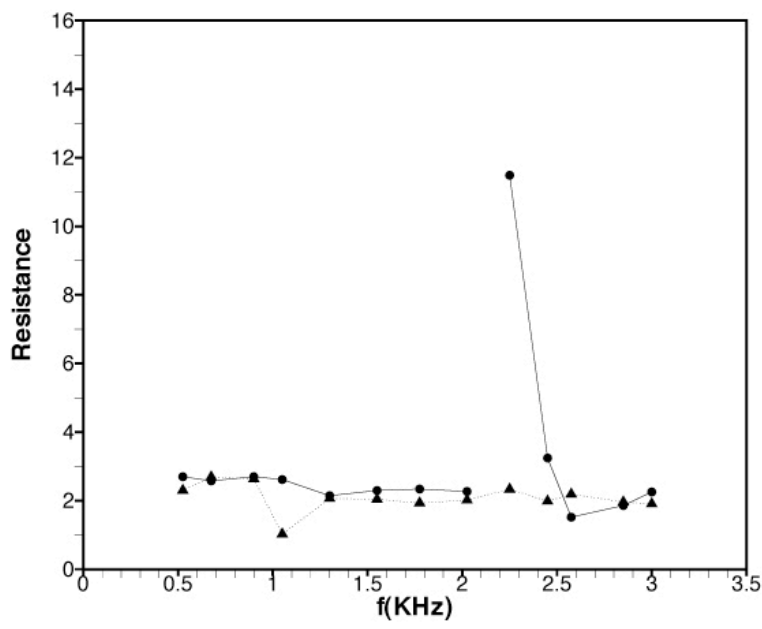

Figure 30. Comparison between calculated and measured normalized resistance for the $45^{\circ}$ slit with 0.05-inch width under pseudo-random acoustic excitation. $\square-$ Experiment, .... ... DNS. 


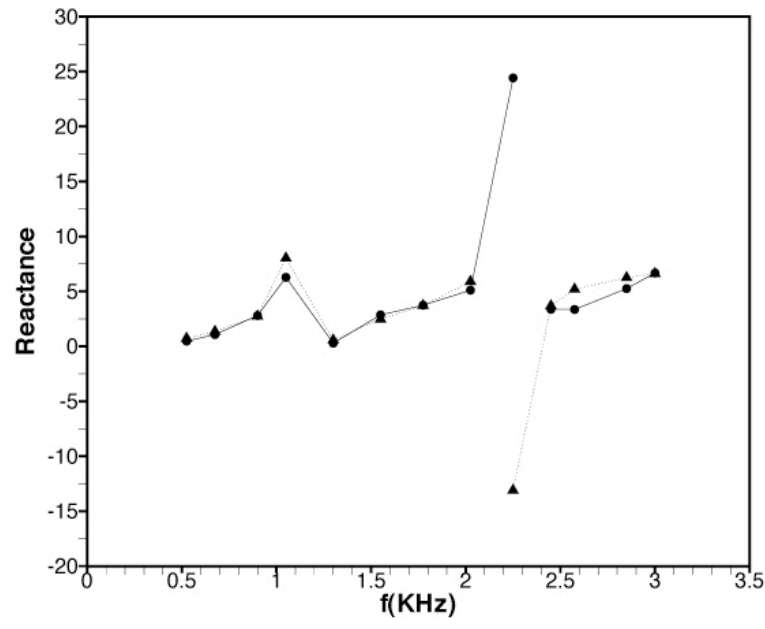

Figure 31. Comparison between calculated and measured normalized reactance for the $45^{\circ}$ slit with 0.05 -inch width under pseudo-random acoustic excitation. $-\square$ Experiment, $\cdots \square \cdots$ DNS.

\section{DNS as a Design Tool}

The purpose of validating DNS results is, of course, to ensure that DNS can offer accurate prediction. However, DNS should not be regarded as only a prediction tool in acoustic liner technology. It has the potential to eventually become a useful design tool. To become an effective tool for design, DNS must overcome a number of hurdles. For example, the effects of multiple slits (or holes), grazing flow, and 3-D (current model is 2-D) must be incorporated. Current acoustic liner simulations require long computer run time. This, however, is not a permanent problem. Future generation fast computers should reduce the run time. The use of massively distributed or parallel computers should also reduce the run time. Improvements on Computational Aeroacoustics (CAA) methodology should also help to shorten significantly the run time required in a simulation.

To illustrate the potential usefulness of DNS as a design tool even under the constraint of current CAA methodology and computer hardware, let us consider the possibility of increasing the resistance of a resonant liner by simple geometrical modification. One simple way to increase the resistance is to enhance vortex shedding at the slit opening. This can be accomplished by using a beveled slit instead of a slit with 90-degree corners. To obtain quantitative data for design consideration, two series of numerical simulations were carried out at identical incident sound levels and frequencies; one for the normal 90-degree slit and the other for the 45-degree bevel slit. The measured acoustic impedance and absorption coefficients are shown in Tables 1 to 3 . Table 1 provides data for the 0.05 -inch slit. Tables 2 and 3 provide similar information for the 0.1 and 0.2-inch slits. These data indicate that except at 0.5 $\mathrm{kHz}$, which is near the resonance frequency of the cavity, the use of a beveled slit increases the resistance. The reason for this increased resistance is simple. A beveled slit promotes vortex shedding that leads to an increase in resistance. Tables 1 to 3 indicate that in a number of cases, there is no vortex shedding associated with the use of a slit with 90degree corners but there is vortex shedding when a bevel slit is used. For these cases, there is an increase in absorption coefficient for the bevel slit. Clearly, as CAA methodology improves such that the hurdles discussed above can be overcome, DNS offers a potential to be a very useful design tool.

It should be noted that continued DNS advances must be validated with measurements of similar quality to those used in the current study. For the current investigation, special effort was taken to match the experimental study to the computational model. For this reason, slit samples were used instead of perforate liners. The geometry of the slit is more amenable to a 2-D fluid dynamics description, whereas the perforated holes of typical liners require a 3D description. 
Table 1

Comparison between the acoustic impedance and absorption coefficients of resonators with 90-degree normal slit and 45-degree bevel slit under identical incident sound pressure level (SPL) and frequency (time dependence $e^{i \square t}$ ).

$N=$ slit with 90-degree corners, $B=45$ degree beveled slit, slit width $=0.05$ inch

\begin{tabular}{|c|c|l|c|c|}
\hline $\begin{array}{c}\text { Freq } \\
\mathrm{kHz}\end{array}$ & $\begin{array}{c}\text { SPL } \\
\mathrm{dB}\end{array}$ & $\begin{array}{c}\text { Vortex } \\
\text { Shedding }\end{array}$ & $\begin{array}{c}\text { Acoustic } \\
\text { Impedance }\end{array}$ & $\begin{array}{c}\text { Absorption } \\
\text { coefficient }\end{array}$ \\
\hline \multirow{2}{*}{0.5} & \multirow{2}{*}{151.1} & $\begin{array}{l}N: \text { strong } \\
B: \text { strong }\end{array}$ & $\begin{array}{c}(3.87833,0.46000) \\
(3.73250,0.66360)\end{array}$ & $\begin{array}{c}0.6452 \\
0.6536\end{array}$ \\
\hline \multirow{2}{*}{1.0} & \multirow{2}{*}{149.9} & $N:$ strong & $(2.15950,4.82325)$ & 0.2599 \\
& & $B:$ strong & $(2.45167,4.63667)$ & 0.2935 \\
\hline \multirow{2}{*}{1.5} & \multirow{2}{*}{150.9} & $N:$ weak & $(2.92275,2.47350)$ & 0.5431 \\
& & $B:$ strong & $(3.24400,2.05967)$ & 0.5832 \\
\hline \multirow{2}{*}{2.0} & \multirow{2}{*}{149.7} & $N:$ weak & $(1.61567,6.58433)$ & 0.1286 \\
& & $B:$ strong & $(1.75650,5.19900)$ & 0.2029 \\
\hline \multirow{2}{*}{2.5} & \multirow{2}{*}{150.0} & $N:$ weak & $(1.83733,5.68667)$ & 0.1821 \\
& & $B:$ strong & $(2.13300,3.87750)$ & 0.3432 \\
\hline \multirow{2}{*}{2.5} & \multirow{2}{*}{142.6} & $N:$ no & $(0.65853,6.40528)$ & 0.0602 \\
& & $B:$ moderate & $(1.07550,4.70200)$ & 0.1629 \\
\hline \multirow{2}{*}{3.0} & \multirow{2}{*}{149.3} & $N:$ no & $(1.25350,9.57025)$ & 0.0519 \\
& & $B:$ weak & $(1.40750,7.32229)$ & 0.0948 \\
\hline \multirow{2}{*}{3.0} & \multirow{2}{*}{141.6} & $N:$ no & $(0.57255,9.59600)$ & 0.0242 \\
& & $B:$ weak & $(0.76897,7.92833)$ & 0.0466 \\
\hline
\end{tabular}

Table 2

slit width $=0.1$ inch

\begin{tabular}{|c|c|c|c|c|}
\hline $\begin{array}{l}\text { Freq } \\
\mathrm{kHz}\end{array}$ & $\begin{array}{c}\text { SPL } \\
\mathrm{dB}\end{array}$ & $\begin{array}{c}\text { Vortex } \\
\text { shedding }\end{array}$ & $\begin{array}{c}\text { Acoustic } \\
\text { impedance }\end{array}$ & $\begin{array}{l}\text { Absorption } \\
\text { coefficient }\end{array}$ \\
\hline 0.5 & 152.7 & $\begin{array}{l}N \text { : strong } \\
B \text { : strong }\end{array}$ & $\begin{array}{l}(2.11700,0.32963) \\
(2.07233,0.38667)\end{array}$ & $\begin{array}{l}0.8611 \\
0.8639\end{array}$ \\
\hline 1.0 & 149.4 & $\begin{array}{l}N \text { : weak } \\
B: \text { weak }\end{array}$ & $\begin{array}{l}(0.49336,4.88040) \\
(0.52075,4.54500)\end{array}$ & $\begin{array}{l}0.0758 \\
0.0907\end{array}$ \\
\hline 1.5 & 151.1 & $\begin{array}{l}N \text { : strong } \\
B \text { : strong }\end{array}$ & $\begin{array}{r}(1.16520,1.97420) \\
(1.20600,1.65567)\end{array}$ & $\begin{array}{l}0.5439 \\
0.6343\end{array}$ \\
\hline 2.0 & 149.2 & $\begin{array}{l}N \text { : weak } \\
B: \text { weak }\end{array}$ & $\begin{array}{l}(0.44830,5.43800) \\
(0.67835,4.85850)\end{array}$ & $\begin{array}{l}0.0566 \\
0.1027\end{array}$ \\
\hline 2.0 & 145.4 & $\begin{array}{l}N: \text { no } \\
B: \text { very weak }\end{array}$ & $\begin{array}{l}(0.24846,5.46192) \\
(0.44803,4.98533)\end{array}$ & $\begin{array}{l}0.0317 \\
0.0665\end{array}$ \\
\hline 2.5 & 151.2 & $\begin{array}{l}N \text { : weak } \\
B: \text { strong }\end{array}$ & $\begin{array}{l}(0.65700,4.07192) \\
(0.93993,3.35367)\end{array}$ & $\begin{array}{l}0.1359 \\
0.2501\end{array}$ \\
\hline 2.5 & 142.7 & $\begin{array}{l}N: \text { no } \\
B: \text { very weak }\end{array}$ & $\begin{array}{l}(0.24263,4.14000) \\
(0.44935,3.56769)\end{array}$ & $\begin{array}{l}0.0519 \\
0.1213\end{array}$ \\
\hline 3.0 & 149.3 & $\begin{array}{l}N: \text { no } \\
B: \text { weak }\end{array}$ & $\begin{array}{l}(0.49213,6.97275) \\
(0.69018,6.24150)\end{array}$ & $\begin{array}{l}0.0387 \\
0.0661\end{array}$ \\
\hline 3.0 & 142.1 & $\begin{array}{l}N: \text { no } \\
B \text { : very weak }\end{array}$ & $\begin{array}{l}(0.22930,6.96100) \\
(0.34117,6.26967)\end{array}$ & $\begin{array}{l}0.0183 \\
0.0332\end{array}$ \\
\hline
\end{tabular}

Table 3

slit width $=0.2$ inch

\begin{tabular}{|c|c|l|c|c|}
\hline $\begin{array}{c}\text { Freq } \\
\mathrm{kHz}\end{array}$ & $\begin{array}{c}\text { SPL } \\
\mathrm{dB}\end{array}$ & $\begin{array}{c}\text { Vortex } \\
\text { shedding }\end{array}$ & $\begin{array}{c}\text { Acoustic } \\
\text { impedance }\end{array}$ & $\begin{array}{c}\text { Absorption } \\
\text { coefficient }\end{array}$ \\
\hline \multirow{2}{*}{0.5} & \multirow{2}{*}{153.8} & $N:$ strong & $(1.03023,0.30539)$ & 0.9685 \\
& & $B:$ strong & $(0.83333,0.26758)$ & 0.9694 \\
\hline \multirow{2}{*}{1.0} & \multirow{2}{*}{149.2} & $N:$ no & $(0.10878,4.38861)$ & 0.0212 \\
& & $B:$ weak & $(0.21879,4.28000)$ & 0.0442 \\
\hline \multirow{2}{*}{1.5} & \multirow{2}{*}{150.6} & $N:$ strong & $(0.40496,1.51300)$ & 0.3797 \\
& & $B:$ strong & $(0.44825,1.32100)$ & 0.4669 \\
\hline \multirow{2}{*}{2.0} & \multirow{2}{*}{149.2} & $N:$ no & $(0.15073,4.15100)$ & 0.0325 \\
& & $B:$ weak & $(0.28870,3.95200)$ & 0.0668 \\
\hline \multirow{2}{*}{2.0} & \multirow{2}{*}{145.2} & $N:$ no & $(0.09041,4.14088)$ & 0.0195 \\
& & $B:$ very weak & $(0.18535,3.93900)$ & 0.0438 \\
\hline \multirow{2}{*}{2.5} & \multirow{2}{*}{149.9} & $N:$ weak & $(0.22483,2.50600)$ & 0.1156 \\
& & $B:$ moderate & $(0.42223,2.28500)$ & 0.2331 \\
\hline \multirow{2}{*}{2.5} & \multirow{2}{*}{142.4} & $N:$ no & $(0.10263,2.49350)$ & 0.0552 \\
& & $B:$ very weak & $(0.21720,2.25800)$ & 0.1319 \\
\hline \multirow{2}{*}{3.0} & \multirow{2}{*}{149.0} & $N:$ no & $(0.19650,5.02733)$ & 0.0294 \\
& & $B:$ weak & $(0.30330,4.71900)$ & 0.0506 \\
\hline \multirow{2}{*}{3.0} & \multirow{2}{*}{142.4} & $N:$ no & $(0.09146,5.01250)$ & 0.0139 \\
& & $B:$ very weak & $(0.15396,4.72000)$ & 0.0261 \\
\hline
\end{tabular}

\section{Concluding Remarks}

In this work, extensive comparisons between experimental measurements and direct numerical simulation results of the interaction of acoustic waves and a slit resonator are calculated. The test matrix includes slits with two different geometries, three slit widths and six discrete sound frequencies. Good agreements are found. Comparisons are also made for broadband incident sound waves with a white noise spectrum and sound waves with a prescribed pseudo-random spectrum. Again good agreements are found. Based on the favorable results, it is our belief that DNS, using advanced CAA methods, has proven to be reliable and accurate for this simple configuration. This offers hope that when using a properly designed computation grid and appropriate mathematical model, DNS has the potential to provide increased understanding of the oscillatory fluid dynamics of orifices in high SPL and high velocity grazing flow environments.

Currently, empirical models are used for liner design and performance prediction. These models are fairly accurate in their domain of application. One of the possible immediate uses of DNS is to try to validate the empirical constants in some of these models 
computationally. If efforts of this kind are successful, it will improve the reliability and accuracy of these models by eliminating a part of their empiricism. Furthermore, by making the older generation model more physics based, their range of applicability might be extended beyond that of their original database.

An important advantage of DNS is that it offers a detailed visualization of the flow and acoustic fields that are usually difficult to obtain experimentally. This is because the slit or hole openings of acoustic liners are generally very small. For this reason, flow visualization has often been conducted on scaled-up models. This approach requires a thorough understanding of all of the issues related with scaling. In Ref.[3], visualization based on the simulation data demonstrated that vortex shedding was the dominant mechanism of acoustic dissipation at high incident sound level. At low SPL, viscous dissipation that took place at two oscillatory shear layers developed slightly off the walls at the opening of the resonator was responsible for the damping of incident sound. This new understanding was made possible by visualization and analysis of DNS data. From this point of view, we regard DNS as a useful complement to laboratory experiment.

In addition to the prediction capability and the possibility of supplementing experimental observations and measurements, DNS has the eventual potential of becoming a useful design tool for acoustic liners. A very simple example is provided in this work. With advances in computer memory and speed, together with advances in CAA methodology, the use of DNS as a design tool may one day become a reality. It is the view of the authors that the results provided in the current report provide a strong foundation for further investigations intended to help us reach this goal.

\section{Acknowledgment}

The support of SP4 computer time by the Florida State University is hereby acknowledged.

\section{References}

1. Ingard, U. and Labate, S, "Acoustic Circulation Effects and the Nonlinear Impedance of Orifics," Journal of the Acoustical Society of America, Vol. 22, 1950, pp. 211-219.

2. Ingard, U. and Ising, H., "Acoustic Nonlinearity of an Orifice," Journal of the Acoustical Society of America, Vol. 42, 1967, pp. 6-17.

3. Tam, C.K.W., and Kurbatskii, K.A., "Microfluid Dynamics and Acoustics of Resonant Liners," AIAA Journal, Vol. 38, No. 8, 2000, pp. 1331-1339.

4. Tam, C.K.W., Kurbatskii, K.A., Ahuja, K.K., and Gaeta, R.J., Jr., "A Numerical and Experimental Investigation of the Dissipation Mechanisms of Resonant Acoustic Liners," Journal of Sound and Vibration, Vol. 245, No. 3, 2001, pp. 545-557.

5. Melling, T.H., "The Acoustic Impedance of Perforates at Medium and High Sound Pressure Levels," Journal of Sound and Vibration, Vol. 29, No. 1, 1973, pp. $1-65$.

6. Zinn, B.T., "A Theoretical Study of Non-Linear Damping by Helmholtz Resonators," Journal of Sound and Vibration, Vol. 13, 1970, pp.347-356.

7. Hersh, A.S. and Walker, B.E., "Acoustic Behavior of Helmholtz Resonators: Part 1, Nonlinear Model," AIAA Paper 95-0078, Jan. 1995.

8. Chung, J.Y. and Blaser, D.A., "Transfer Function Method of Measuring In-duct Acoustic Properties: I. Theory," Journal of Acoustical Society of America, Vol. 68, 1980, pp. 907-921.

9. Jones, M.G. and Parrott, T.L., "Evaluation of a Multi-Point Method for Determining Acoustic Impedance," Journal of Mechanical Systems and Signal Processing, Vol. 3, No. 1, 1989, pp. 15-35.

10. White, F.M., Viscous Fluid Flow. (Chapter 3), $2^{\text {nd }}$ edition, McGraw Hill, 1991.

11. Tam, C.K.W. and Webb, J.C., "Dispersion-RelationPreserving Finite Difference Schemes for Computational Acoustics," Journal of Computational Physics, Vol. 17, Aug. 1993, pp. 262-281.

12. Tam, C.K.W., "Computational Aeroacoustics: Issues and Methods," AIAA Journal, Vol. 33, No. 10, 1995, pp. 1788-1796.

13. Tam, C.K.W. and Kurbatskii, K.A., "Multi-Size-Mesh Multi-Time-Step Dispersion-Relation-Preserving Scheme for Multiple-Scales Aeroacoustics problems," International Journal of Computational Fluid Dynamics, Vol. 17, No. 2, 2003, pp. 119-132.

14. Tam, C.K.W., Webb, J.C. and Dong, Z., "A Study of the Short Wave Components in Computational Acoustics," Journal of Computational Acoustics, Vol. 1, March 1993, pp.1-30. 